\title{
Real group orbits on flag manifolds
}

\author{
Dmitri Akhiezer
}

\begin{abstract}
In this survey, we gather together various results on the action of a real form $G_{0}$ of a complex semisimple group $G$ on its flag manifolds. We start with the finiteness theorem of J. Wolf implying that at least one of the $G_{0}$-orbits is open. We give a new proof of the converse statement for real forms of inner type, essentially due to F.M. Malyshev. Namely, if a real form of inner type $G_{0} \subset G$ has an open orbit on a complex algebraic homogeneous space $G / H$ then $H$ is parabolic. In order to prove this, we recall, partly with proofs, some results of A.L. Onishchik on the factorizations of reductive groups. Finally, we discuss the cycle spaces of open $G_{0}$-orbits and define the crown of a symmetric space of noncompact type. With some exceptions, the cycle space agrees with the crown. We sketch a complex analytic proof of this result, due to G. Fels, A. Huckleberry and J. Wolf.
\end{abstract}

\section{Introduction}

The first systematic treatment of the orbit structure of a complex flag manifold $X=G / P$ under the action of a real form $G_{0} \subset G$ is due to J. Wolf [38]. Forty years after his paper, these real group orbits and their cycle spaces are still an object of intensive research. We present here some results in this area, together with other related results on transitive and locally transitive actions of Lie groups on complex manifolds.

The paper is organized as follows. In Section 2 we prove the celebrated finiteness theorem for $G_{0}$-orbits on $X$ (Theorem 2.3). We also state a theorem characterizing open $G_{0}$-orbits on $X$ (Theorem 2.4). All results of Section 2 are taken from [38]. In Section 3 we recall for future use a theorem, due to B. Weisfeiler [36] and A. Borel and J. Tits [4]. Namely, let $H$ be an algebraic subgroup of a connected reductive group $G$. Theorem 3.1 shows that one can find a parabolic subgroup $P \subset G$ containing $H$, such that the unipotent radical of $H$ is contained in the unipotent radical of $P$. In Section 4 we consider the factorizations of reductive groups. The results of this section are due to A.L. Onishchik [30, 31]. We take for granted his list of factorizations $G=H_{1} \cdot H_{2}$, where $G$ is a simple algebraic group over $\mathbb{C}$ and $H_{1}, H_{2} \subset G$ are reductive complex subgroups (Theorem 4.1), and deduce from it his theorem on real forms. Namely, a 
real form $G_{0}$ acting locally transitively on an affine homogeneous space $\mathrm{G} / \mathrm{H}$ is either $\mathrm{SO}_{1,7}$ or $\mathrm{SO}_{3,5}$. Moreover, in that case $\mathrm{G} / \mathrm{H}=\mathrm{SO}_{8} / \mathrm{Spin}_{7}$ and the action of $G_{0}$ is in fact transitive (Corollary 4.7). This very special homogeneous space of a complex group $G$ has on open orbit of a real form $G_{0}$, the situation being typical for flag manifolds. One can ask what homogeneous spaces share this property. It turns out that if a real form of inner type $G_{0} \subset G$ has an open orbit on a homogeneous space $G / H$ with $H$ algebraic, then $H$ is in fact parabolic and so $G / H$ is a flag manifold. We prove this in Section 5 (see Corollary 5.2) and then retrieve the result of F.M. Malyshev of the same type in which the isotropy subgroup is not necessarily algebraic (Theorem 5.4). It should be noted that, the other way around, the statement for algebraic homogeneous spaces can be deduced from his theorem. Our proof of both results is new.

Let $K$ be the complexification of a maximal compact subgroup $K_{0} \subset G_{0}$. In Section 6 we briefly recall the Matsuki correspondence between $G_{0}$ - and $K$-orbits on a flag manifold. In Section 7 we define, following the paper of S.G. Gindikin and the author [1], the crown $\Xi$ of $G_{0} / K_{0}$ in $G / K$. We also introduce the cycle space of an open $G_{0}$-orbit on $X=G / P$, first considered by R. Wells and J. Wolf [37], and state a theorem describing the cycle spaces in terms of the crown (Theorem 7.1). In fact, with some exceptions which are well-understood, the cycle space of an open $G_{0}$-orbit on $X$ agrees with $\Xi$ and, therefore, is independent of the flag manifold. In Sections 8 and 9 , we give an outline of the original proof due to G. Fels, A. Huckleberry and J. Wolf [11], using the methods of complex analysis. One ingredient of the proof is a theorem of G. Fels and A. Huckleberry [10], saying that $\Xi$ is a maximal $G_{0}$-invariant, Stein and Kobayashi hyperbolic domain in $G / K$ (Theorem 8.4). Another ingredient is the construction of the Schubert domain, due to A. Huckleberry and J. Wolf [16] and explained in Section 9. Finally, in Section 10 we discuss complex geometric properties of flag domains. Namely, let $q$ be the dimension of the compact $K$-orbit in an open $G_{0}$-orbit. We consider measurable open $G_{0}$-orbits and state the theorem of W. Schmid and J. Wolf [33] on the $q$-completeness of such flag domains.

Given a $K$-orbit $O$ and the corresponding $G_{0}$-orbit $O^{\prime}$ on $X$, S.G. Gindikin and T. Matsuki suggested to consider the subset $C(O) \subset G$ of all $g \in G$, such that $g O \cap O^{\prime} \neq \emptyset$ and $g O \cap O^{\prime}$ is compact, see [13]. If $O$ is compact then $O^{\prime}$ is open and $O \subset O^{\prime}$. Furthermore, in this case $C(O)=\{g \in$ $\left.G \mid g O \subset O^{\prime}\right\}$ is precisely the set whose connected component $C(O)^{\circ}$ at $e \in G$ is the cycle space of $O^{\prime}$ lifted to $G$. This gives a natural way of generalizing the notion of a cycle space to lower-dimensional $G_{0}$-orbits. Recently, using this generalization, T. Matsuki carried over Theorem 7.1 to arbitrary $G_{0}$-orbits on flag manifolds, see [27] and Theorem 7.2. His proof is beyond the scope of our survey. 


\section{Finiteness theorem}

Let $G$ be a connected complex semisimple Lie group, $\mathfrak{g}$ the Lie algebra of $G$, and $\mathfrak{g}_{0}$ a real form of $\mathfrak{g}$. The complex conjugation of $\mathfrak{g}$ over $\mathfrak{g}_{0}$ is denoted by $\tau$. Let $G_{0}$ be the connected real Lie subgroup of $G$ with Lie algebra $\mathfrak{g}_{0}$. We are interested in $G_{0}$-orbits on flag manifolds of $G$. By definition, these manifolds are the quotients of the form $G / P$, where $P \subset G$ is a parabolic subgroup. It is known that the intersection of two parabolic subgroups in $G$ contains a maximal torus of $G$. Equivalently, the intersection of two parabolic subalgebras in $\mathfrak{g}$ contains a Cartan subalgebra of $\mathfrak{g}$. We want to prove a stronger statement in the case when the parabolic subalgebras are $\tau$-conjugate. We will use the notion of a Cartan subalgebra for an arbitrary (and not just semisimple) Lie algebra $\mathfrak{l}$ over any field $k$. Recall that a Lie subalgebra $\mathfrak{j} \subset \mathfrak{l}$ is called a Cartan subalgebra if $\mathfrak{j}$ is nilpotent and equal to its own normalizer. Given a field extension $k \subset k^{\prime}$, it follows from that definition that $\mathfrak{j}$ is a Cartan subalgebra in $\mathfrak{l}$ if and only if $\mathbf{j} \otimes_{k} k^{\prime}$ is a Cartan subalgebra in $\mathfrak{l} \otimes_{k} k^{\prime}$. We start with a simple general observation.

Lemma 2.1. Let $\mathfrak{g}$ be any complex Lie algebra, $\mathfrak{g}_{0}$ a real form of $\mathfrak{g}$, and $\tau: \mathfrak{g} \rightarrow \mathfrak{g}$ the complex conjugation of $\mathfrak{g}$ over $\mathfrak{g}_{0}$. Let $\mathfrak{h} \subset \mathfrak{g}$ be a complex Lie subalgebra. Then $\mathfrak{h} \cap \mathfrak{g}_{0}$ is a real form of $\mathfrak{h} \cap \tau(\mathfrak{h})$.

Proof. For any $A \in \mathfrak{h} \cap \tau(\mathfrak{h})$ one has $2 A=(A+\tau(A))+(A-\tau(A))$, where the first summand is contained in $\mathfrak{h} \cap \mathfrak{g}_{0}$ and the second one gets into that subspace after multiplication by $i$.

The following corollary will be useful.

Corollary 2.2. If $\mathfrak{p}$ is a parabolic subalgebra of a semisimple algebra $\mathfrak{g}$, then $\mathfrak{p} \cap \tau(\mathfrak{p})$ contains a $\tau$-stable Cartan subalgebra $\mathfrak{t}$ of $\mathfrak{g}$.

Proof. Choose a Cartan subalgebra $\mathfrak{j}$ of $\mathfrak{p} \cap \mathfrak{g}_{0}$. Its complexification $\mathfrak{t}$ is a Cartan subalgebra of $\mathfrak{p} \cap \tau(\mathfrak{p})$, which is $\tau$-stable. Now, $\mathfrak{p} \cap \tau(\mathfrak{p})$ contains a Cartan subalgebra $\mathfrak{t}^{\prime}$ of $\mathfrak{g}$. Since $\mathfrak{t}$ and $\mathfrak{t}^{\prime}$ are conjugate as Cartan subalgebras of $\mathfrak{p} \cap \tau(\mathfrak{p})$, it follows that $\mathfrak{t}$ is itself a Cartan subalgebra of $\mathfrak{g}$.

The number of conjugacy classes of Cartan subalgebras of a real semisimple Lie algebra is finite. This was proved independently by A. Borel and B. Kostant in the fifties of the previous century, see [18]. Somewhat later, M. Sugiura determined explicitly the number of conjugacy classes and found their representatives for each simple Lie algebra, see [34]. Let $\left\{\mathfrak{j}_{1}, \ldots, \mathfrak{j}_{m}\right\}$ be a complete system of representatives of Cartan subalgebras of $\mathfrak{g}_{0}$. For each $k, k=1, \ldots, m$, the complexification $\mathfrak{t}_{k}$ of $\mathfrak{j}_{k}$ is a Cartan subalgebra of $\mathfrak{g}$.

Theorem 2.3 (J. Wolf [38], Thm. 2.6). For any parabolic subgroup $P \subset G$ the number of $G_{0}$-orbits on $X=G / P$ is finite.

Proof. Define a map $\iota: X \rightarrow\{1, \ldots, m\}$ as follows. For any point $x \in X$ let $\mathfrak{p}_{x}$ be the isotropy subalgebra of $x$ in $\mathfrak{g}$. By Corollary 2.2, we can choose a Cartan subalgebra $\mathfrak{j}_{x}$ of $\mathfrak{g}_{0}$ in $\mathfrak{p}_{x}$. Take $g \in G_{0}$ so that $\operatorname{Ad} g \cdot \mathfrak{j}_{x}=\mathfrak{j}_{k}$ 
for some $k, k=1, \ldots, m$. Since $\mathfrak{j}_{k}$ and $\mathfrak{j}_{l}$ are not conjugate for $k \neq l$, the number $k$ does not depend on $g$. Let $k=\iota(x)$. Then $\iota(x)$ is constant along the orbit $G_{0}(x)$. Now, for $\iota(x)$ fixed there exists $g \in G_{0}$ such that $\mathfrak{p}_{g x}$ contains $\mathfrak{t}_{k}$ with fixed $k$. Recall that a point of $X$ is uniquely determined by its isotropy subgroup. Since there are only finitely many parabolic subgroups containing a given maximal torus, the fiber of $\iota$ has finitely many $G_{0}$-orbits.

As a consequence of Theorem 2.3 , we see that at least one $G_{0}$-orbit is open in $X$. We will need a description of open orbits in terms of isotropy subalgebras of their points. Fix a Cartan subalgebra $\mathfrak{t} \subset \mathfrak{g}$. Let $\Sigma=\Sigma(\mathfrak{g}, \mathfrak{t})$ be the root system, $\mathfrak{g}_{\alpha} \subset \mathfrak{g}, \alpha \in \Sigma$, the root subspaces, $\Sigma^{+}=\Sigma^{+}(\mathfrak{g}, \mathfrak{t}) \subset \Sigma$ a positive subsystem, and $\Pi$ the set of simple roots corresponding to $\Sigma^{+}$. Every $\alpha \in \Sigma$ has a unique expression $\alpha=\sum_{\pi \in \Pi} n_{\pi}(\alpha) \cdot \pi$, where $n_{\pi}(\alpha)$ are integers, all non-negative for $\alpha \in \Sigma^{+}$and all non-positive for $\alpha \in \Sigma^{-}=-\Sigma^{+}$. For an arbitrary subset $\Phi \subset \Pi$ we will use the notation

$$
\Phi^{r}=\left\{\alpha \in \Sigma \mid n_{\pi}(\alpha)=0 \text { whenever } \pi \notin \Phi\right\}, \quad \Phi^{u}=\left\{\alpha \in \Sigma^{+} \mid \alpha \notin \Phi^{r}\right\} .
$$

Then the standard parabolic subalgebra $\mathfrak{p}_{\Phi} \subset \mathfrak{g}$ is defined by

$$
\mathfrak{p}_{\Phi}=\mathfrak{p}_{\Phi}^{r}+\mathfrak{p}_{\Phi}^{u}
$$

where

$$
\mathfrak{p}_{\Phi}^{r}=\mathfrak{t}+\sum_{\alpha \in \Phi^{r}} \mathfrak{g}_{\alpha} \text { and } \mathfrak{p}_{\Phi}^{u}=\sum_{\alpha \in \Phi^{u}} \mathfrak{g}_{\alpha}
$$

are the standard reductive Levi subalgebra and, respectively, the unipotent radical of $\mathfrak{p}_{\Phi}$. In the sequel, we will also use the notation

$$
\mathfrak{p}_{\Phi}^{-u}=\sum_{-\alpha \in \Phi^{u}} \mathfrak{g}_{\alpha}
$$

Now, let $\mathfrak{k}_{0}$ be a maximal compact subalgebra of $\mathfrak{g}_{0}$. Then we have the Cartan involution $\theta: \mathfrak{g}_{0} \rightarrow \mathfrak{g}_{0}$ and the Cartan decomposition $\mathfrak{g}_{0}=\mathfrak{k}_{0}+\mathfrak{m}_{0}$, where $\mathfrak{k}_{0}$ and $\mathfrak{m}_{0}$ are the eigenspaces of $\theta$ with eigenvalues 1 and, respectively, -1. A $\theta$-stable Cartan subalgebra $\mathfrak{j} \subset \mathfrak{g}_{0}$ is called fundamental (or maximally compact) if $\mathfrak{j} \cap \mathfrak{k}_{0}$ is a Cartan subalgebra of $\mathfrak{k}_{0}$. More generally, a Cartan subalgebra $\mathfrak{j} \subset \mathfrak{g}_{0}$ is called fundamental if $\mathfrak{j}$ is conjugate to a $\theta$-stable fundamental Cartan subalgebra. It is known that any two fundamental Cartan subalgebras of $\mathfrak{g}_{0}$ are conjugate under an inner automorphism of $\mathfrak{g}_{0}$. We will assume that a Cartan subalgebra $\mathfrak{t} \subset \mathfrak{g}$ is $\tau$-stable. In other words, $\mathfrak{t}=\mathfrak{j}^{\mathbb{C}}$, where $\mathfrak{j}$ is a Cartan subalgebra in $\mathfrak{g}_{0}$. Then $\tau$ acts on $\Sigma$ by $\tau(\alpha)(A)=\overline{\alpha(\tau \cdot A)}$, where $\alpha \in \Sigma, A \in \mathfrak{t}$.

Theorem 2.4 (J. Wolf [38], Thm.4.5). Let $X=G / P$ be a flag manifold. Then the $G_{0}$-orbit of $x_{0}=e \cdot P$ is open in $X$ if and only if $\mathfrak{p}=\mathfrak{p}_{\Phi}$, where

(i) $\mathfrak{p} \cap \mathfrak{g}_{0}$ contains a fundamental Cartan subalgebra $\mathfrak{j} \subset \mathfrak{g}_{0}$;

(ii) $\Phi$ is a subset of simple roots for $\Sigma^{+}(\mathfrak{g}, \mathfrak{t}), \mathfrak{t}=\mathfrak{j}^{\mathbb{C}}$, such that $\tau \Sigma^{+}=$ $\Sigma^{-}$

The proof can be also found in [11], Sect. 4.2. 


\section{Embedding a subgroup into a parabolic one}

Let $G$ be a group. The normalizer of a subgroup $H \subset G$ is denoted by $N_{G}(H)$. For an algebraic group $H$ the unipotent radical is denoted by $R_{u}(H)$.

Let $U$ be an algebraic unipotent subgroup of a complex semisimple group $G$. Set $N_{1}=N_{G}(U), U_{1}=R_{u}\left(N_{1}\right)$, and continue inductively:

$$
N_{k}=N_{G}\left(U_{k-1}\right), \quad U_{k}=R_{u}\left(N_{k}\right), \quad k \geq 2 .
$$

Then $U \subset U_{1}$ and $U_{k-1} \subset U_{k}, \quad N_{k-1} \subset N_{k}$ for all $k \geq 2$. Therefore there exists an integer $l$, such that $U_{l}=U_{l+1}$. This means that $U_{l}$ coincides with the unipotent radical of its normalizer. We now recall the following general theorem of fundamental importance.

Theorem 3.1 (B. Weisfeiler [36], A. Borel, J. Tits [4], Cor. 3.2). Let $k$ be an arbitrary field, $G$ a connected reductive algebraic group defined over $k$, and $U$ a unipotent algebraic subgroup of $G$. If the unipotent radical of the normalizer $N_{G}(U)$ coincides with $U$, then $N_{G}(U)$ is a parabolic subgroup of $G$.

For $k=\mathbb{C}$, which is the only case we need, the result goes back to V.V. Morozov, see [4], Remarque 3.4. In the above form, the theorem was conjectured by I.I. Piatetski-Shapiro, see [36]. For future references, we state the following corollary of Theorem 3.1.

Corollary 3.2. Let $k=\mathbb{C}$ and let $G$ be as above. The normalizer $N_{G}(U)$ of a unipotent algebraic subgroup $U \subset G$ embeds into a parabolic subgroup $P \subset G$ in such a way that $U \subset R_{u}(P)$. For any algebraic subgroup $H \subset G$ there exists an embedding into a parabolic subgroup $P$, such that $R_{u}(H) \subset R_{u}(P)$.

Proof. Put $P=N_{G}\left(U_{l}\right)$ in the above construction. Then $U \subset U_{l}=$ $R_{u}(P)$. This proves the first assertion. To prove the second one, it suffices to take $U=R_{u}(H)$.

\section{Factorizations of reductive groups}

The results of this section are due to A.L. Onishchik. Let $G$ be a group, $H_{1}, H_{2} \subset G$ two subgroups. A triple $\left(G ; H_{1}, H_{2}\right)$ is called a factorization of $G$ if for any $g \in G$ there exist $h_{1} \in H_{1}$ and $h_{2} \in H_{2}$, such that $g=h_{1} \cdot h_{2}$. In the Lie group case a factorization $\left(G ; H_{1}, H_{2}\right)$ gives rise to the factorization $\left(\mathfrak{g} ; \mathfrak{h}_{1}, \mathfrak{h}_{2}\right)$ of the Lie algebra $\mathfrak{g}$. By definition, this means that $\mathfrak{g}=\mathfrak{h}_{1}+\mathfrak{h}_{2}$. Conversely, if $\left(\mathfrak{g} ; \mathfrak{h}_{1}, \mathfrak{h}_{2}\right)$ is a factorization of $\mathfrak{g}$ then the product $H_{1} \cdot H_{2}$ is an open subset in $G$ containing the neutral element. In general, this open set does not coincide with $G$, and so a factorization $\left(\mathfrak{g} ; \mathfrak{h}_{1}, \mathfrak{h}_{2}\right)$ is sometimes called a local factorization of $G$. But, if $G, H_{1}$ and $\mathrm{H}_{2}$ are connected reductive (complex or real) Lie groups then every local 
factorization is (induced by) a global one, see [31]. We will give a simple proof of this fact below, see Prop. 4.3 and Prop. 4.4.

All factorizations of connected compact Lie groups are classified in [30], see also [32], $\S 14$. If $G, H_{1}$ and $H_{2}$ are connected reductive (complex or real) Lie groups, then the same problem is solved in [31]. The core of the classification is the complete list of factorizations for simple compact Lie groups. We prefer to state the result for simple algebraic groups over $\mathbb{C}$. If both subgroups $H_{1}, H_{2}$ are reductive algebraic, then the list is the same as in the compact case.

Theorem 4.1 (A.L. Onishchik $[30,31])$. If $G$ is a simple algebraic group over $k=\mathbb{C}$ and $H_{1}, H_{2}$ are proper reductive algebraic subgroups of $G$, then, up to a local isomorphism and renumbering of factors, the factorization $\left(G ; H_{1}, H_{2}\right)$ is one of the following:

(1) $\left(S L_{2 n} ; S p_{2 n}, S L_{2 n-1}\right), n \geq 2$;

(2) $\left(S L_{2 n} ; S p_{2 n}, S\left(G L_{1} \times G L_{2 n-1}\right), n \geq 2\right.$;

(3) $\left(\mathrm{SO}_{7} ; \mathrm{G}_{2}, \mathrm{SO}_{6}\right)$;

(4) $\left(\mathrm{SO}_{7} ; \mathrm{G}_{2}, \mathrm{SO}_{5}\right)$;

(5) $\left.\mathrm{SO}_{7} ; \mathrm{G}_{2}, \mathrm{SO}_{3} \times \mathrm{SO}_{2}\right)$;

(6) $\left(S O_{2 n} ; S O_{2 n-1}, S L_{n}\right), n \geq 4$

(7) $\left(S O_{2 n} ; S O_{2 n-1}, G L_{n}\right), n \geq 4$;

(8) $\left(S O_{4 n} ; S O_{4 n-1}, S p_{2 n}\right), n \geq 2$;

(9) $\left(S O_{4 n} ; S O_{4 n-1}, S p_{2 n} \cdot S p_{2}\right), n \geq 2$;

(10) $\left(S O_{4 n} ; S O_{4 n-1}, S p_{2 n} \cdot k^{*}\right), n \geq 2$;

(11) $\left(S O_{16} ; S O_{15}\right.$, Spin $\left._{9}\right)$;

(12) $\left(\mathrm{SO}_{8} ; \mathrm{SO}_{7}, \mathrm{Spin}_{7}\right)$.

Although this result is algebraic by its nature, the only known proof uses topological methods. We want to show how Theorem 4.1 applies to factorizations of complex Lie algebras involving their real forms.

Lemma 4.2. Let $\tau: \mathfrak{g} \rightarrow \mathfrak{g}$ be the complex conjugation of a complex Lie algebra over its real form $\mathfrak{g}_{0}$. Let $\mathfrak{h} \subset \mathfrak{g}$ be a complex Lie subalgebra. Then $\mathfrak{g}=\mathfrak{g}_{0}+\mathfrak{h}$ if and only if $\mathfrak{g}=\mathfrak{h}+\tau(\mathfrak{h})$.

Proof. Let $\mathfrak{g}=\mathfrak{g}_{0}+\mathfrak{h}$. For any $X \in \mathfrak{g}_{0}$ one has $i X=Y+Z$, where $Y \in \mathfrak{g}_{0}$ and $Z \in \mathfrak{h}$. This implies

$$
2 X=-i Z-\tau(i Z) \in \mathfrak{h}+\tau(\mathfrak{h}) .
$$

Conversely, if $\mathfrak{g}=\mathfrak{h}+\tau(\mathfrak{h})$, then for any $X \in \mathfrak{g}$ there exist $Z_{1}, Z_{2} \in \mathfrak{h}$, such that

$$
X=Z_{1}+\tau\left(Z_{2}\right)=\left(Z_{1}-Z_{2}\right)+\left(Z_{1}+\tau\left(Z_{2}\right)\right),
$$

hence $X \in \mathfrak{h}+\mathfrak{g}_{0}$.

Proposition 4.3. Let $G$ be a connected reductive algebraic group over $\mathbb{C}$ and let $H_{1}, H_{2} \subset G$ be two reductive algebraic subgroups. Then $\mathfrak{g}=\mathfrak{h}_{1}+\mathfrak{h}_{2}$ if and only if $\mathrm{G}=\mathrm{H}_{1} \cdot \mathrm{H}_{2}$. 
Proof. It suffices to prove that the local factorization implies the global one. Let $X=G / H_{2}$ and let $n=\operatorname{dim}(X)$. If $L$ is a maximal compact subgroup of $H_{2}$ and $K$ is a maximal compact subgroup of $G$, such that $L \subset$ $K$, then $X$ is diffeomorphic to a real vector bundle over $K / L$. Therefore $X$ is homotopically equivalent to a compact manifold of (real) dimension $n$. On the other hand, $H_{1}$ has an open orbit on $X$. Since $X$ is an affine variety, closed $H_{1}$-orbits are separated by $H_{1}$-invariant regular functions. But such functions are constant, so there is only one closed orbit. Assume now that $H_{1}$ is not transitive on $X$, so that the closed $H_{1}$-orbit has dimension $m<n$. A well-known corollary of Luna's Slice Theorem displays $X$ as a vector bundle over the closed orbit, see [21]. Thus $X$ is homotopically equivalent to that orbit and, by the same argument as above, to a compact manifold of (real) dimension $m$. Now, for a compact connected manifold $M$ of dimension $n$ one has $H_{i}\left(M, \mathbb{Z}_{2}\right)=0$ if $i>n$ and $H_{n}\left(M, \mathbb{Z}_{2}\right) \cong \mathbb{Z}_{2}$, see e.g. [9], Prop. 3.3 and Cor. 3.4. Therefore two compact manifolds of dimensions $m$ and $n, m \neq n$, are not homotopically equivalent, and we get a contradiction.

As a corollary, we have a similar proposition for real groups.

Proposition 4.4. Let $G, H_{1}$ and $H_{2}$ be real forms of complex reductive algebraic groups $G^{\mathbb{C}}, H_{1}^{\mathbb{C}}$ and $H_{2}^{\mathbb{C}}$. For $G$ connected one has $\mathfrak{g}=\mathfrak{h}_{1}+\mathfrak{h}_{2}$ if and only if $\mathrm{G}=\mathrm{H}_{1} \cdot \mathrm{H}_{2}$.

Proof. If $\mathfrak{g}=\mathfrak{h}_{1}+\mathfrak{h}_{2}$ then $\mathfrak{g}^{\mathbb{C}}=\mathfrak{h}_{1}{ }^{\mathbb{C}}+\mathfrak{h}_{2}{ }^{\mathbb{C}}$. Thus $G^{\mathbb{C}}=H_{1}^{\mathbb{C}} \cdot H_{2}^{\mathbb{C}}$ by Proposition 4.3. The action of $H_{1}^{\mathbb{C}} \times H_{2}^{\mathbb{C}}$ on $G^{\mathbb{C}}$, defined by

$$
g \mapsto h_{1} g h_{2}^{-1}, \quad g \in G^{\mathbb{C}}, h_{i} \in H_{i}^{\mathbb{C}},
$$

is transitive. For $g \in G \subset G^{\mathbb{C}}$ we have the following estimate of the dimension of $\left(H_{1} \times H_{2}\right)$-orbit through $g$ :

$$
\operatorname{dim} H_{1} g H_{2}=\operatorname{dim} H_{1}+\operatorname{dim} H_{2}-\operatorname{dim}\left(H_{1} \cap g H_{2} g^{-1}\right) \geq
$$

$$
\operatorname{dim}_{\mathbb{C}} H_{1}^{\mathbb{C}}+\operatorname{dim}_{\mathbb{C}} H_{2}^{\mathbb{C}}-\operatorname{dim}_{\mathbb{C}} H_{1}^{\mathbb{C}} \cap g H_{2}^{\mathbb{C}} g^{-1}=\operatorname{dim}_{\mathbb{C}} G^{\mathbb{C}}=\operatorname{dim} G .
$$

But $G$ is connected and each coset $H_{1} g H_{2}$ is open, hence $G=H_{1} \cdot H_{2}$.

We will use the notion of an algebraic subalgebra of a complex Lie algebra $\mathfrak{g}$, which corresponds to an algebraic group $G$. A subalgebra $\mathfrak{h} \subset \mathfrak{g}$ is said to be algebraic, if the associated connected subgroup $H \subset G$ is algebraic. In general, this notion depends on the choice of $G$. However, if $\mathfrak{g}$ is semisimple, which will be our case, then $\mathfrak{h}$ is algebraic for some $G$ if and only if $\mathfrak{h}$ is algebraic for any other $G$. An algebraic subalgebra of $\mathfrak{g}$ is said to be reductive, if $H$ is a reductive algebraic subgroup of $G$. Again, for $\mathfrak{g}$ semisimple the choice of $G$ does not matter.

Theorem 4.5 (cf. [31], Thm. 4.2). Let $\mathfrak{g}$ be a simple complex Lie algebra, $\mathfrak{h} \subset \mathfrak{g}, \mathfrak{h} \neq \mathfrak{g}$, a reductive algebraic subalgebra, and $\mathfrak{g}_{0}$ a real form of $\mathfrak{g}$. If $\mathfrak{g}=\mathfrak{g}_{0}+\mathfrak{h}$ then $\mathfrak{g}$ is of type $\mathrm{D}_{4}, \mathfrak{h}$ is of type $\mathrm{B}_{3}$, embedded as the spinor subalgebra, and $\mathfrak{g}_{0}$ is either $\mathfrak{s o}_{1,7}$ or $\mathfrak{s o}_{3,5}$. 
Proof. In the notation of Lemma 4.2 we have $\mathfrak{g}=\mathfrak{h}+\tau(\mathfrak{h})$. Note that $\tau(\mathfrak{h})$ is a reductive algebraic subalgebra of $\mathfrak{g}$. Choose $G$ simply connected. Then $\tau$ lifts to an antiholomorphic involution of $G$, which we again denote by $\tau$. Let $H_{1}$ and $H_{2}$ be the connected reductive algebraic subgroups of $G$ with Lie algebras $\mathfrak{h}$ and, respectively, $\tau(\mathfrak{h})$. By Proposition 4.3 we have the global decomposition $G=H_{1} \cdot H_{2}$. Since $H_{1}$ and $H_{2}$ are isomorphic, it follows from Theorem 4.1 that the factorization $\left(G ; H_{1}, H_{2}\right)$ is obtained from factorization (12). More precisely, $G$ is isomorphic to $\operatorname{Spin}_{8}$, the universal covering group of $\mathrm{SO}_{8}$, and $\mathrm{H}_{1}, \mathrm{H}_{2}$ are two copies of $\operatorname{Spin}_{7}$ in $\operatorname{Spin}_{8}$. We assume that $H_{1}$ is the image of the spinor representation $\mathrm{Spin}_{7} \rightarrow \mathrm{SO}_{8}$ and $\mathrm{H}_{2}$ comes from the embedding $\mathrm{SO}_{7} \rightarrow \mathrm{SO}_{8}$. The conjugation $\tau$ interchanges $H_{1}$ and $H_{2}$. We want to replace $\tau$ by a holomorphic involutive automorphism of $G$ with the same behaviour with respect to $H_{1}$ and $H_{2}$. For this we need the following lemma.

Lemma 4.6. Let $G$ be a connected reductive algebraic group over $\mathbb{C}$. Take a maximal compact subgroup in $G$ which is invariant under $\tau$. Let $\theta$ : $G \rightarrow G$ be the corresponding Cartan involution and let $\sigma=\theta \tau(=\tau \theta)$. For a reductive algebraic subgroup $H \subset G$, the factorization $(G ; H, \tau(H))$ implies the factorization $(G ; H, \sigma(H))$, and vice versa.

Proof. First of all, if $\left(G ; H_{1}, H_{2}\right)$ is a factorization of a group then one also has the factorization $\left(G ; \tilde{H}_{1}, \tilde{H}_{2}\right)$, where $\tilde{H}_{1}=g_{1} H_{1} g_{1}^{-1}, \tilde{H}_{2}=g_{2} H_{2} g_{2}^{-1}$ are conjugate subgroups. In the setting of the lemma, choose $\tilde{H}=g \mathrm{Hg}^{-1}$ so that a maximal compact subgroup of $\tilde{H}$ is contained in the chosen maximal compact subgroup of $G$. Then $\theta(\tilde{H})=\tilde{H}$ and, consequently,

$$
\tau(H) \simeq \tau(\tilde{H})=\sigma(\tilde{H}) \simeq \sigma(H),
$$

where $\simeq$ denotes conjugation by an inner automorphism. By the above remark, one of the two factorizations $(G ; H, \tau(H)),(G ; H, \sigma(H))$ implies the other.

End of proof of Theorem 4.5. We can replace $H_{2}$ by a conjugate subgroup so that $H_{1}$ and $H_{2}$ are interchanged by $\sigma$. The factorization is in fact defined for $\mathrm{SO}_{8}$, in which case the subgroups are only locally isomorphic. For this reason, $\sigma$ is an outer automorphism. It follows that the restriction of $\sigma$ to the real form, i.e., the Cartan involution of the latter, is also an outer automorphism. There are precisely two real forms of $\mathrm{D}_{4}$ with this property, namely, $\mathfrak{s o}_{1,7}$ and $\mathfrak{s o}_{3,5}$. The remaining non-compact real forms $\mathfrak{s o}_{2,6}, \mathfrak{s o}_{4,4}$, and $\mathfrak{s o}_{8}^{*}$ are of inner type, see Sect 5 . We still have to show that $\mathfrak{s o}_{1,7}$, as well as $\mathfrak{s o}_{3,5}$, together with the complex spinor subalgebra, gives a factorization of $\mathfrak{g}=\mathfrak{s o}_{8}$. So let $\tau$ be the complex conjugation of $\mathfrak{g}$ over $\mathfrak{s o}_{1,7}$ or $\mathfrak{s o}_{3,5}$. Define $\sigma$ as in the lemma and denote again by $\sigma$ the corresponding automorphism of $\mathfrak{g}$. The fixed point subalgebra of $\sigma$ has rank 3, whereas $\mathfrak{g}$ has rank 4 . Thus $\sigma$ is an outer automorphism of $\mathfrak{g}$. There are three conjugacy classes of subalgebras of type $\mathrm{B}_{3}$ in $\mathfrak{g}$. Let $\Upsilon$ be the set of these conjugacy classes. The group of outer isomorphisms of $\mathfrak{g}$ acts on $\Upsilon$ as the group of all permutations of $\Upsilon$, isomorphic to the symmetric group $S_{3}$. Choose $\mathcal{C} \in \Upsilon$ so that $\sigma(\mathcal{C}) \neq \mathcal{C}$ and let $\mathfrak{h} \in \mathcal{C}$. 
Applying an outer automorphism of $\mathfrak{g}$, we can arrange that $\mathfrak{h}$ corresponds to $\operatorname{Spin}_{7}$ and $\sigma(\mathfrak{h})=\mathfrak{s o}_{7}$. Therefore $\mathfrak{g}=\mathfrak{h}+\sigma(\mathfrak{h})$ by Theorem 4.1 and $\mathfrak{g}=\mathfrak{g}_{0}+\mathfrak{h}$ by Lemma 4.6 and Lemma 4.2.

Corollary 4.7. Let $G$ be a simple algebraic group over $\mathbb{C}, G_{0}$ a real form of $G$, and $H \subset G$ a proper reductive algebraic subgroup. Then the following three conditions are equivalent:

(i) $G_{0}$ is locally transitive on $G / H$;

(ii) $G_{0}$ is transitive on $G / H$;

(iii) up to a local isomorphism, $G=S O_{8}, H=\operatorname{Spin}_{7}, G_{0}=S O_{1,7}$ or $\mathrm{SO}_{3,5}$.

Proof. Theorem 4.5 says that (i) and (iii) are equivalent. Proposition 4.4 shows that (i) implies (ii).

\section{Real forms of inner type}

Let $\mathfrak{g}_{0}$ be a real semisimple Lie algebra of non-compact type. Let $\mathfrak{g}_{0}=\mathfrak{k}_{0}+$ $\mathfrak{m}_{0}$ be a Cartan decomposition with the corresponding Cartan involution $\theta$. It is known that $\theta$ is an inner automorphism of $\mathfrak{g}_{0}$ if and only if $\mathfrak{k}_{0}$ contains a Cartan subalgebra of $\mathfrak{g}_{0}$. If this is the case, we will say that the Lie algebra $\mathfrak{g}_{0}$ and the corresponding Lie group $G_{0}$ is of inner type. Clearly, $\mathfrak{g}_{0}$ is of inner type if and only if all simple ideals of $\mathfrak{g}_{0}$ are of inner type. The Cartan classification yields the following list of simple Lie algebras of inner type:

$$
\mathfrak{s l}_{2}(\mathbb{R}), \mathfrak{s u}_{p, q}, \mathfrak{s o}_{p, q}(p \text { or } q \text { even }), \mathfrak{s o}_{2 n}^{*}, \mathfrak{s p}_{2 n}(\mathbb{R}), \mathfrak{s p}_{p, q}
$$

\section{EII, EIII, EV, EVI, EVII, EVIII, EIX, FI, FII, G.}

As we have seen in Sect.1, a conjugacy class of parabolic subalgebras has a representative $\mathfrak{p}$, such that $\mathfrak{g}=\mathfrak{g}_{0}+\mathfrak{p}$. In other words, for any parabolic subgroup $P \subset G$ the real form $G_{0}$ has an open orbit on $G / P$. For real forms of inner type the converse is also true.

Theorem 5.1. Let $\mathfrak{g}$ be a complex semisimple Lie algebra, $\mathfrak{g}_{0}$ a real form of $\mathfrak{g}$ of inner type, and $\mathfrak{j}$ a compact Cartan subalgebra of $\mathfrak{g}_{0}$. If $\mathfrak{h}$ is an algebraic Lie subalgebra of $\mathfrak{g}$ satisfying $\mathfrak{g}=\mathfrak{g}_{0}+\mathfrak{h}$ then $\mathfrak{h}$ is parabolic. Moreover, there exists an inner automorphism $\operatorname{Ad}(g), g \in G_{0}$, such that $\mathfrak{h}=\operatorname{Ad}(g) \cdot \mathfrak{p}_{\Phi}$, where $\Phi$ is a subset of simple roots for some ordering of $\Sigma\left(\mathfrak{g}, \mathfrak{j}^{\mathbb{C}}\right)$. Conversely, any such $\mathfrak{h}$ satisfies $\mathfrak{g}=\mathfrak{g}_{0}+\mathfrak{h}$.

Corollary 5.2. Let $G$ be a complex semisimple Lie group, $G_{0} \subset G$ a real form of inner type, and $H \subset G$ a complex algebraic subgroup. If $G_{0}$ has an open orbit on $G / H$ then $H$ is parabolic.

For an algebraic Lie algebra $\mathfrak{h}$ we denote by $R_{u}(\mathfrak{h})$ the unipotent radical and by $L(\mathfrak{h})$ a reductive Levi subalgebra. For the proof of Theorem 5.1 we will need a lemma which rules out certain factorizations with semisimple factors. 
Lemma 5.3. Let $\mathfrak{g}$ be a simple complex Lie algebra and let $\mathfrak{h}_{1}, \mathfrak{h}_{2} \subset \mathfrak{g}$ be two semisimple real Lie subalgebras, such that $\mathfrak{h}_{1} \cap \mathfrak{h}_{2}=\{0\}$. Then $\mathfrak{g} \neq \mathfrak{h}_{1}+\mathfrak{h}_{2}$.

Proof. Assume $\mathfrak{h}_{1}+\mathfrak{h}_{2}=\mathfrak{g}$. Let $G$ be a simply connected Lie group with Lie algebra $\mathfrak{g}$ and let $H_{1}, H_{2}$ be connected subgroups of $G$ with Lie algebras $\mathfrak{h}_{1}, \mathfrak{h}_{2}$. Then $G=H_{1} \cdot H_{2}$ by Prop. 4.4. Therefore one can write $G$ as a homogeneous space $G=\left(H_{1} \times H_{2}\right) /\left(H_{1} \cap H_{2}\right)$, where $H_{1} \cap H_{2}$ embeds diagonally into the product. Because $G$ is simply connected, we see that the intersection $H_{1} \cap H_{2}$ is in fact trivial. But $H^{3}(G, \mathbb{R}) \cong \mathbb{R}$, whereas $\operatorname{dim} H^{3}\left(H_{i}, \mathbb{R}\right) \geq 1$, see e.g. [32], Ch. $3, \S 9$, and so the decomposition $G=H_{1} \times H_{2}$ yields a contradiction.

Proof of Theorem 5.1. Write $\mathfrak{g}_{0}$ as the sum of simple ideals $\mathfrak{g}_{k, 0}, k=$ $1, \ldots, m$. Each of them is stable under the Cartan involution $\theta$, because $\theta$ is an inner automorphism. Furthermore, each $\mathfrak{g}_{k, 0}$ is again of inner type. Thus the complexification $\mathfrak{g}_{k}=\left(\mathfrak{g}_{k, 0}\right)^{\mathbb{C}}$ is a simple ideal of $\mathfrak{g}$, and $\mathfrak{g}=\mathfrak{g}_{1} \oplus \ldots \oplus \mathfrak{g}_{m}$. Let $\pi_{k}: \mathfrak{g} \rightarrow \mathfrak{g}_{k}$ be the projection map.

Assume that $\mathfrak{h}$ is reductive. We want to show that then $\mathfrak{h}=\mathfrak{g}$. For each $k$ we have $\mathfrak{g}_{k}=\mathfrak{g}_{k, 0}+\pi_{k}(\mathfrak{h})$. If $\pi_{k}(\mathfrak{h}) \neq \mathfrak{g}_{k}$, then $\mathfrak{g}_{k, 0}$ is isomorphic to $\mathfrak{s o}_{1,7}$ or $\mathfrak{s o}_{3,5}$ by Corollary 4.7. Since $\mathfrak{g}_{k, 0}$ is of inner type, this can not happen. Hence $\pi_{k}(\mathfrak{h})=\mathfrak{g}_{k}$ for all $k$. In particular, $\mathfrak{h}$ is semisimple, and so we write $\mathfrak{h}$ as the sum of simple ideals $\mathfrak{h}=\mathfrak{h}_{1} \oplus \ldots \oplus \mathfrak{h}_{n}$. Since $\pi_{k}\left(\mathfrak{h}_{l}\right)$ is an ideal in $\mathfrak{g}_{k}$, there are only two possibilities: $\pi_{k}\left(\mathfrak{h}_{l}\right)=\mathfrak{g}_{k}$ or $\pi_{k}\left(\mathfrak{h}_{l}\right)=\{0\}$. If, for $k$ fixed, we have $\pi_{k}\left(\mathfrak{h}_{l}\right) \neq\{0\}$ and $\pi_{k}\left(\mathfrak{h}_{s}\right) \neq\{0\}$ then in fact $s=l$, because otherwise

$$
\mathfrak{g}_{k}=\left[\mathfrak{g}_{k}, \mathfrak{g}_{k}\right]=\left[\pi_{k}\left(\mathfrak{h}_{l}\right), \pi_{k}\left(\mathfrak{h}_{s}\right)\right]=\pi_{k}\left(\left[\mathfrak{h}_{l}, \mathfrak{h}_{s}\right]\right)=\{0\} .
$$

We want to make sure that $m=n$. In that case, renumbering the simple ideals of $\mathfrak{g}$, we get $\mathfrak{h}_{l} \subset \mathfrak{g}_{l}$ for all $l$. This implies $\mathfrak{h}_{l}=\mathfrak{g}_{l}$ for all $l$ and $\mathfrak{h}=\mathfrak{g}$. Now, if $n<m$ then one and only one $\mathfrak{h}_{l}$ projects isomorphically onto $\mathfrak{g}_{k}$ and $\mathfrak{g}_{p}$ for $p \neq k$. Let $\omega_{k}=\left(\pi_{k} \mid \mathfrak{h}_{l}\right)^{-1}$ and $\omega_{p}=\left(\pi_{p} \mid \mathfrak{h}_{l}\right)^{-1}$. Then

$$
\mathfrak{g}_{k} \oplus \mathfrak{g}_{p}=\left(\pi_{k} \oplus \pi_{p}\right)\left(\mathfrak{h}_{l}\right)+\left(\mathfrak{g}_{k, 0} \oplus \mathfrak{g}_{p, 0}\right),
$$

hence

$$
\mathfrak{h}_{l}=\omega_{k}\left(\mathfrak{g}_{k, 0}\right)+\omega_{p}\left(\mathfrak{g}_{p, 0}\right),
$$

and so a simple complex Lie algebra $\mathfrak{h}_{l}$ is written as the sum of two real forms. This contradicts Lemma 5.3.

Assume from now on that $R_{u}(\mathfrak{h}) \neq\{0\}$ and take an embedding of $\mathfrak{h}$ into a parabolic subalgebra $\mathfrak{p}$, such that $R_{u}(\mathfrak{h}) \subset R_{u}(\mathfrak{p})$, see Cor. 3.2. Then $\mathfrak{g}=\mathfrak{g}_{0}+\mathfrak{p}$, i.e., the $G_{0}$-orbit of the base point is open in $G / P$. By Theorem $2.4 \mathfrak{p}$ is a standard parabolic subalgebra, $\mathfrak{p}=\mathfrak{p}_{\Phi}$, where:

(i) $\mathfrak{p} \cap \mathfrak{g}_{0}$ contains a fundamental Cartan subalgebra $\mathfrak{j} \subset \mathfrak{g}_{0}$, which is now compact (recall that $\mathfrak{g}_{0}$ is of inner type);

(ii) $\Phi$ is a subset of simple roots for some ordering of $\Sigma(\mathfrak{g}, \mathfrak{t}), \mathfrak{t}=\mathfrak{j}^{\mathbb{C}}$ (since $\mathfrak{j}$ is compact, $\tau(\alpha)=-\alpha$ for all $\alpha \in \Sigma(\mathfrak{g}, \mathfrak{t})$ and $\tau \Sigma^{+}=\Sigma^{-}$for any choice of $\Sigma^{+}$). 
By our construction, $R_{u}(\mathfrak{h}) \subset \mathfrak{p}_{\Phi}^{u}$. Applying an inner automorphism of $\mathfrak{p}_{\Phi}$, assume that $L(\mathfrak{h}) \subset \mathfrak{p}_{\Phi}^{r}$. Since $\tau\left(\mathfrak{g}_{\alpha}\right)=\mathfrak{g}_{-\alpha}$ for all root spaces, we have

$$
\tau\left(\mathfrak{p}_{\Phi}^{r}\right)=\mathfrak{p}_{\Phi}^{r} \text { and } \tau\left(\mathfrak{p}_{\Phi}^{u}\right)=\mathfrak{p}_{\Phi}^{-u} .
$$

Observe that $\tau(\mathfrak{h})+\mathfrak{h}=\mathfrak{g}$ by Lemma 4.2. Therefore

$$
\tau\left(R_{u}(\mathfrak{h})\right)+R_{u}(\mathfrak{h})=\tau\left(\mathfrak{p}_{\Phi}^{u}\right)+\mathfrak{p}_{\Phi}^{u}=\mathfrak{p}_{\Phi}^{-u}+\mathfrak{p}_{\Phi}^{u},
$$

and so we obtain

$$
R_{u}(\mathfrak{h})=\mathfrak{p}_{\Phi}^{u}
$$

We also have

$$
\tau(L(\mathfrak{h}))+L(\mathfrak{h})=\mathfrak{p}_{\Phi}^{r},
$$

hence, again by Lemma 4.2 ,

$$
\mathfrak{p}_{\Phi}^{r}=\left(\mathfrak{p}_{\Phi}^{r}\right)_{0}+L(\mathfrak{h}), \quad \text { where } \quad\left(\mathfrak{p}_{\Phi}^{r}\right)_{0}=\mathfrak{p}_{\Phi}^{r} \cap \mathfrak{g}_{0} .
$$

Write $\mathfrak{p}_{\Phi}^{r}=\mathfrak{s}+\mathfrak{z}$, where $\mathfrak{s}$ is the semisimple part and $\mathfrak{z}$ the center of $\mathfrak{p}_{\Phi}^{r}$, denote by $\pi_{s}, \pi_{z}$ the corresponding projections, and put $\mathfrak{s}_{0}=\mathfrak{s} \cap \mathfrak{g}_{0}, \mathfrak{z}_{0}=$ $\mathfrak{z} \cap \mathfrak{g}_{0}$. Then $\mathfrak{s}=\mathfrak{s}_{0}+\pi_{s}(L(\mathfrak{h}))$. Since $\mathfrak{s}_{0}$ is a semisimple algebra of inner type and $\pi_{s}(L(\mathfrak{h}))$ is reductive, we get $\pi_{s}(L(\mathfrak{h}))=\mathfrak{s}$ by what we have already proved. Therefore $L(\mathfrak{h})=\mathfrak{s}+\mathfrak{z}_{*}$, where $\mathfrak{z}_{*}$ is an algebraic subalgebra in $\mathfrak{z}$. On the other hand, $\mathfrak{z}=\mathfrak{z}_{0}+\pi_{z}(L(\mathfrak{h}))=\mathfrak{z}_{0}+\mathfrak{z}_{*}$. But $\mathfrak{z}_{0}$ is compact, so $\mathfrak{z}_{*}=\mathfrak{z}$ and $L(\mathfrak{h})=\mathfrak{p}_{\Phi}^{r}$. Together with the equality $R_{u}(\mathfrak{h})=$ $\mathfrak{p}_{\Phi}^{u}$, this gives $\mathfrak{h}=\mathfrak{p}_{\Phi}$. To finish the proof, recall that any two compact Cartan subalgebras of $\mathfrak{g}_{0}$ are conjugate by an inner automorphism. For the converse statement of the theorem, note that, $\mathfrak{j}$ being compact, (ii) in Theorem 2.4 is fulfilled for any ordering of $\Sigma(\mathfrak{g}, \mathfrak{t})$.

We now recover a theorem of F.M. Malyshev, in which $\mathfrak{h}$ is not necessarily algebraic. Of course, our Theorem 5.1 is a special case of his result. We want to show that the general case can be obtained from that special one. We adopt the notation introduced in the above proof. Namely, $\mathfrak{s}=\mathfrak{s} \Phi$ and $\mathfrak{z}=\mathfrak{z}_{\Phi}$ are the semisimple part and, respectively, the center of the reductive algebra $\mathfrak{p}_{\Phi}^{r}$.

Theorem 5.4 (F.M. Malyshev [22]). Let $\mathfrak{g}, \mathfrak{g}_{0}$ and $\mathfrak{j}$ be as in Theorem 5.1. If $\mathfrak{h}$ is a complex Lie subalgebra of $\mathfrak{g}$ satisfying $\mathfrak{g}=\mathfrak{g}_{0}+\mathfrak{h}$ then there exists an inner automorphism $\operatorname{Ad}(g), g \in G_{0}$, such that $\mathfrak{h}=\operatorname{Ad}(g)\left(\mathfrak{a}+\mathfrak{s}_{\Phi}+\mathfrak{p}_{\Phi}^{u}\right)$, where $\Phi$ is a subset of simple roots for some ordering of $\Sigma\left(\mathfrak{g}, \mathfrak{j}^{\mathbb{C}}\right)$ and $\mathfrak{a}$ is a complex subspace of $\mathfrak{z}_{\Phi}$ which projects onto the real form $\left(\mathfrak{z}_{\Phi}\right)_{0}$. Conversely, any such $\mathfrak{h}$ satisfies $\mathfrak{g}=\mathfrak{g}_{0}+\mathfrak{h}$.

Proof. Let $\mathfrak{h}_{\text {alg }}$ be the algebraic closure of $\mathfrak{h}$, i.e., the smallest algebraic subalgebra of $\mathfrak{g}$ containing $\mathfrak{h}$. According to a theorem of C. Chevalley, the commutator algebras of $\mathfrak{h}$ and $\mathfrak{h}_{\text {alg }}$ are the same, see [8]. Chap. II, Théorème 13. Applying an inner automorphism $\operatorname{Ad}(g), g \in G_{0}$, we get

$$
\mathfrak{h}_{\text {alg }}=\mathfrak{p}_{\Phi}=\mathfrak{z}_{\Phi}+\mathfrak{s}_{\Phi}+\mathfrak{p}_{\Phi}^{u}
$$


by Theorem 5.1. Since $\mathfrak{h}$ contains $\left[\mathfrak{h}_{\text {alg }}, \mathfrak{h}_{\text {alg }}\right]=\mathfrak{s}_{\Phi}+\mathfrak{p}_{\Phi}^{u}$, it follows that

$$
\mathfrak{h}=\mathfrak{a}+\mathfrak{s}_{\Phi}+\mathfrak{p}_{\Phi}^{u}
$$

where $\mathfrak{a} \subset \mathfrak{z}_{\Phi}$ is a complex subspace. Observe that

$$
\tau(\mathfrak{h})=\tau(\mathfrak{a})+\mathfrak{s}_{\Phi}+\mathfrak{p}_{\Phi}^{-u} .
$$

By Lemma 4.2 we have $\mathfrak{g}=\mathfrak{h}+\tau(\mathfrak{h})$. Clearly, $\mathfrak{z}_{\Phi}$ is $\tau$-stable. The above expression for $\tau(\mathfrak{h})$ shows that $\mathfrak{z}_{\Phi}=\mathfrak{a}+\tau(\mathfrak{a})$. Again by Lemma 4.2, this implies $\mathfrak{z}_{\Phi}=\left(\mathfrak{z}_{\Phi}\right)_{0}+\mathfrak{a}$ or, equivalently, $\mathfrak{z}_{\Phi}=i \cdot\left(\mathfrak{z}_{\Phi}\right)_{0}+\mathfrak{a}$. Thus a projects onto $\left(\mathfrak{z}_{\Phi}\right)_{0}$. Since $\tau \mathfrak{p}_{\Phi}^{u}=\mathfrak{p}_{\Phi}^{-u}$, the converse statement is obvious.

If $\mathfrak{g}_{0}$ is a real form of outer type (= not of inner type), then a Lie subalgebra $\mathfrak{h} \subset \mathfrak{g}$, satisfying $\mathfrak{g}=\mathfrak{g}_{0}+\mathfrak{h}$, is in general very far from being parabolic. Some classification of such $\mathfrak{h}$ is known for type $\mathrm{D}_{n}$, see [23]. Here is a typical example of what can happen for other Lie algebras.

Example 5.5. Let $\mathfrak{g}=\mathfrak{s l}_{2 n}(\mathbb{C}), n>1$, and let $\tau(A)=\bar{A}$ for $A \in \mathfrak{g}$, so that $\mathfrak{g}_{0}=\mathfrak{s l}_{2 n}(\mathbb{R})$. Then there is a fundamental Cartan subalgebra $\mathfrak{j} \subset \mathfrak{g}_{0}$ and an ordering of the root system $\Sigma(\mathfrak{g}, \mathfrak{t}), \mathfrak{t}=\mathfrak{j}^{\mathbb{C}}$, such that the set of simple roots $\Pi$ is of the form $\Pi=\Phi \sqcup \Psi \sqcup\{\gamma\}$, where $\Phi$ and $\Psi$ are orthogonal, $\tau(\Phi)=-\Psi$ and $\tau(\gamma)=-\gamma$. The standard Levi subalgebra of $\mathfrak{p}_{\Phi \sqcup \Psi}$ can be written as

$$
\mathfrak{p}_{\Phi \sqcup \Psi}^{r}=\mathfrak{s}_{1}+\mathfrak{s}_{2}+\mathfrak{z}
$$

where $\mathfrak{s}_{1}$ and $\mathfrak{s}_{2}$ are isomorphic simple algebras of type $A_{n-1}$ interchanged by $\tau$ and $\mathfrak{z}$ is a $\tau$-stable one-dimensional torus. Set

$$
\mathfrak{h}=\mathfrak{s}_{1}+\mathfrak{z}+\mathfrak{p}_{\Phi \sqcup \Psi}^{u},
$$

then

$$
\tau(\mathfrak{h})=\mathfrak{s}_{2}+\mathfrak{z}+\mathfrak{p}_{\Phi \sqcup \Psi}^{-u} .
$$

Therefore $\mathfrak{h}+\tau(\mathfrak{h})=\mathfrak{g}$, showing that $\mathfrak{g}=\mathfrak{g}_{0}+\mathfrak{h}$. Note that $\mathfrak{h}$ is an ideal in the parabolic subalgebra $\mathfrak{p}=\mathfrak{p}_{\Phi \sqcup \Psi}$, such that $\mathfrak{p} / \mathfrak{h}$ is a simple algebra.

The construction of $\mathfrak{j}$ and the ordering in $\Sigma(\mathfrak{g}, \mathfrak{t})$ goes as follows. Take the Cartan decomposition $\mathfrak{g}_{0}=\mathfrak{k}_{0}+\mathfrak{m}_{0}$, where $\mathfrak{k}_{0}=\mathfrak{s o}_{2 n}(\mathbb{R})$. Define $\mathfrak{j}$ as the space of block matrices

$$
\left(\begin{array}{ccccccc}
a_{1} & b_{1} & & & & & \\
-b_{1} & a_{1} & & & & & \\
& & a_{2} & b_{2} & & & \\
& & -b_{2} & a_{2} & & & \\
& & & & \ddots & & \\
& & & & & a_{n} & b_{n} \\
& & & & & -b_{n} & a_{n}
\end{array}\right)
$$

with real entries and $\Sigma a_{i}=0$. Then $\mathfrak{j}$ is a fundamental Cartan subalgebra and $\mathfrak{j}=\mathfrak{j} \cap \mathfrak{k}_{0}+\mathfrak{j} \cap \mathfrak{m}_{0}$. Consider $a_{i}$ and $b_{i}$ as linear functions on $\mathfrak{j}$ and 
$\mathfrak{t}=\mathfrak{j}^{\mathbb{C}}$. Then it is easy to determine the root system $\Sigma(\mathfrak{g}, \mathfrak{t})$. We list the roots that we declare positive:

$i\left(b_{p}-b_{q}\right) \pm\left(a_{p}-a_{q}\right), i\left(b_{p}+b_{q}\right) \pm\left(a_{p}-a_{q}\right)(p<q)$, and $2 i b_{p} \quad(p, q=1, \ldots, n)$.

Let $\Phi=\left\{\alpha_{1}, \ldots, \alpha_{n-1}\right\}, \Psi=\left\{\beta_{1}, \ldots, \beta_{n-1}\right\}$, where

$\alpha_{p}=i\left(b_{p}-b_{p+1}\right)+a_{p}-a_{p+1}, \beta_{p}=i\left(b_{p}-b_{p+1}\right)-a_{p}+a_{p+1}(p=1, \ldots, n-1)$,

and let $\gamma=2 i b_{n}$. Then the set of simple roots $\Pi$ is the union $\Pi=$ $\Phi \sqcup \Psi \sqcup\{\gamma\}, \Phi$ and $\Psi$ are orthogonal, $\tau\left(\alpha_{p}\right)=-\beta_{p}$ for all $p$ and $\tau(\gamma)=-\gamma$.

\section{Matsuki correspondence}

Recall that $G_{0}$ is a real form of a complex semisimple group $G$ and both $G_{0}$ and $G$ are connected. Let $\mathfrak{g}_{0}=\mathfrak{k}_{0}+\mathfrak{m}_{0}$ be a Cartan decomposition, $\mathfrak{k}$ the complexification in $\mathfrak{g}$ of $\mathfrak{k}_{0}$, and $K$ the corresponding connected reductive subgroup of $G$.

Theorem 6.1 (T. Matsuki [25]). Let $O$ be a $K$-orbit and let $O^{\prime}$ be a $G_{0}$-orbit on $G / P$, where $P \subset G$ is a parabolic subgroup. The relation

$$
O \leftrightarrow O^{\prime} \Longleftrightarrow O \cap O^{\prime} \neq \emptyset \text { and } O \cap O^{\prime} \text { is compact }
$$

defines a bijection between $K \backslash G / P$ and $G_{0} \backslash G / P$.

A geometric proof of this result, using the moment map technique, is found in [28], [5]. Note that $K$ is a spherical subgroup of $G$, i.e., a Borel subgroup of $G$ has an open orbit on $G / K$. It that case $B$ has finitely many orbits on $G / K$, see [6], [35]. Thus the set $K \backslash G / P$ is finite, and so $G_{0} \backslash G / P$ is also finite (another proof of Theorem 2.3).

It can happen that both $K \backslash G / P$ and $G_{0} \backslash G / P$ are one-point sets. For $G$ simple, there are only two types of such actions.

Theorem 6.2 (A.L. Onishchik [31], Thm. 6.1). If $G$ is simple and $G_{0}$ or, equivalently, $K$ is transitive on $X=G / P$ then, up to a local isomorphism,

(1) $G=S L_{2 n}(\mathbb{C}), K=S p_{2 n}(\mathbb{C}), G_{0}=S U_{2 n}^{*}, X=\mathbb{P}^{2 n-1}(\mathbb{C})$, or

$(2) G=S O_{2 n}(\mathbb{C}), K=S O_{2 n-1}(\mathbb{C}), G_{0}=S O_{2 n-1,1}^{o}, X=S O_{2 n}(\mathbb{R}) / U_{n}$.

There are two important cases of the correspondence $O \leftrightarrow O^{\prime}$, namely, when one of the two orbits is open or when it is compact. The first of the following two propositions is evident, and the second one is due to T. Matsuki [24].

Proposition 6.3. If $O$ is open then $O^{\prime}$ is compact and $O^{\prime} \subset O$. If $O^{\prime}$ is open then $O$ is compact and $O \subset O^{\prime}$.

Proposition 6.4. If $O$ is compact then $O^{\prime}$ is open and $O \subset O^{\prime}$. If $O^{\prime}$ is compact then $O$ is open and $O^{\prime} \subset O$. 
Proof. We prove the second statement. The proof of the first one is similar. Take a base point $x_{0} \in O \cap O^{\prime}$ and let $P$ be the isotropy subgroup of $x_{0}$. Note that $G_{0} \cap P$ has only finitely many connected components, since it is an open subgroup of a real algebraic group. By a theorem of D. Montgomery [29], $K_{0}$ is transitive on the compact homogeneous space $G_{0} /\left(G_{0} \cap P\right)$, hence

$$
\mathfrak{g}_{0}=\mathfrak{k}_{0}+\mathfrak{g}_{0} \cap \mathfrak{p} \subset \mathfrak{k}+\mathfrak{p} .
$$

On the other hand, $\mathfrak{k}_{0}+i \mathfrak{m}_{0}$ is the Lie algebra of a maximal compact subgroup of $G$, which is transitive on $G / P$. Therefore

$$
\mathfrak{g}=\mathfrak{k}_{0}+i \mathfrak{m}_{0}+\mathfrak{p}
$$

or, equivalently

$$
\mathfrak{g}=i \mathfrak{k}_{0}+\mathfrak{m}_{0}+\mathfrak{p}
$$

and it follows that $\mathfrak{g} \subset \mathfrak{k}+\mathfrak{g}_{0}+\mathfrak{p} \subset \mathfrak{k}+\mathfrak{p}$, i.e., $\mathfrak{g}=\mathfrak{k}+\mathfrak{p}$. This means that $O=K\left(x_{0}\right)$ is open in $G / P$, and the inclusion $O^{\prime} \subset O$ follows from Proposition 6.3.

\section{Cycle spaces}

First, we recall the definition of the complex crown of a real symmetric space $G_{0} / K_{0}$, see [1]. Let $\mathfrak{a} \subset \mathfrak{m}_{0}$ be a maximal abelian subspace and let $\mathfrak{a}^{+} \subset \mathfrak{a}$ be the subset given by the inequalities $|\alpha(Y)|<\frac{\pi}{2}$, where $Y \in \mathfrak{a}$ and $\alpha$ runs over all restricted roots, i. e., the roots of $\mathfrak{g}_{0}$ with respect to $\mathfrak{a}$. Then the crown is the set

$$
\Xi=G_{0}\left(\exp i \mathfrak{a}^{+}\right) o \subset G / K,
$$

where $o=e \cdot K \in G / K$ is the base point. The set $\Xi$ is open and the $G_{0^{-}}$ action on $\Xi$ is proper, see [1]. We discuss some properties of the complex manifold $\Xi$ in the next section. Because all maximal abelian subspaces in $\mathfrak{m}_{0}$ are $K_{0}$-conjugate, it follows that $\Xi$ is independent of the choice of $\mathfrak{a}$ and is therefore determined by $G_{0} / K_{0}$ itself. Some authors call $\Xi$ the universal domain, see [11]. We reserve this term for the lift of $\Xi$ to $G$ and define the universal domain by

$$
\Omega=G_{0}\left(\exp i \mathfrak{a}^{+}\right) K \subset G,
$$

due to the properties of $\Omega$ which will soon become clear. Of course, $\Omega$ is invariant under the right $K$-action and $\Omega / K=\Xi$.

Next, we define the (linear) cycle space for an open $G_{0}$-orbit on $X=G / P$, see [37]. Since full cycle spaces (in the sense of D.Barlet) are not discussed here, we will omit the adjective "linear". Let $D$ be such an orbit and let $C_{0}$ be the corresponding $K$-orbit, so that if $O \leftrightarrow O^{\prime}$ for $O=C_{0}$ and $O^{\prime}=D$. The orbit $C_{0}$ is a compact complex manifold contained in $D$. Consider the open set

$$
G\{D\}=\left\{g \in G \mid g C_{0} \subset D\right\} \subset G
$$


and denote by $G\{D\}^{\circ}$ its connected component containing $e \in G$. Observe that $G\{D\}$ is invariant under the right multiplication by $L=\{g \in$ $\left.G \mid g C_{0}=C_{0}\right\}$ and left multiplication by $G_{0}$. Since $L$ is a closed complex subgroup of $G$, we have a natural complex structure on $G / L$. By definition, the cycle space $\mathcal{M}_{D}$ of $D$ is the connected component of $C_{0}(=e \cdot L)$ in $G\{D\} / L$ with the inherited $G_{0}$-invariant complex structure.

In what follows, we assume $\mathfrak{g}$ simple. We will say that $G_{0}$ is of Hermitian type if the symmetric space $G_{0} / K_{0}$ is Hermitian. If this is the case then $\mathfrak{g}$ has three irreducible components as $(\operatorname{ad} \mathfrak{k})$-module, namely, $\mathfrak{g}=\mathfrak{s}^{-}+\mathfrak{k}+\mathfrak{s}^{+}$, where $\mathfrak{s}^{+}, \mathfrak{s}^{-}$are abelian subalgebras. The subalgebras $\mathfrak{k}+\mathfrak{s}^{+}$and $\mathfrak{k}+\mathfrak{s}^{-}$are parabolic. The corresponding parabolic subgroups are denoted $P^{+}$and $P^{-}$. We have two flag manifolds $X^{+}=$ $G / P^{+}, X^{-}=G / P^{-}$with base points $x^{+}=e \cdot P^{+}, x^{-}=e \cdot P^{-}$and two $G_{0}$-invariant complex structures on $G_{0} / K_{0}$ defined by the equivariant embeddings $g \cdot K_{0} \mapsto g x^{ \pm} \in X^{ \pm}$. Each of the two orbits $\mathcal{B}=G_{0}\left(x^{+}\right)$and $\overline{\mathcal{B}}=G_{0}\left(x^{-}\right)$is biholomorphically isomorphic to the bounded symmetric domain associated to $G_{0}$. The Lie algebra $\mathfrak{l}$ of $L$ contains $\mathfrak{k}$. If $G_{0}$ is of Hermitian type and $\mathfrak{l}$ coincides with $\mathfrak{p}^{+}$or $\mathfrak{p}^{-}$then we say that $D$ and, also, the corresponding compact $K$-orbit $C_{0}$ is of (Hermitian) holomorphic type. If $G_{0}$ is of non-Hermitian type then $\mathfrak{k}$ is a maximal proper subalgebra of $\mathfrak{g}$. Thus, if $\mathfrak{l} \neq \mathfrak{g}$ then $\mathfrak{l}=\mathfrak{k}$. For $G_{0}$ of Hermitian type, each flag manifold has exactly two $K$-orbits of holomorphic type. All other $K$ orbits for $G_{0}$ of Hermitian type and all $K$-orbits for $G_{0}$ of non-Hermitian type are said to be of non-holomorphic type. In the following theorem, we exclude the actions listed in Theorem 6.2. The symbol $\simeq$ means a $G_{0}$-equivariant biholomorphic isomorphism.

Theorem 7.1 (G. Fels, A. Huckleberry, J.A. Wolf [11], Thm. 11.3.7). Assume $G$ simple and suppose $G_{0}$ is not transitive on $X=G / P$. Let $D$ be an open $G_{0}$-orbit on $X$. If $D$ is of holomorphic type then $\mathcal{M}_{D} \simeq \mathcal{B}$ or $\mathcal{M}_{D} \simeq \overline{\mathcal{B}}$. In all other cases, $G\{D\}^{\circ}$ coincides with the universal domain $\Omega \subset G$. Moreover, $\pi: G / K \rightarrow G / L$ is a finite covering map, which induces a $G_{0}$-equivarint biholomorphic map $\left.\pi\right|_{\Xi}: \Xi \rightarrow \mathcal{M}_{D}$.

If $G_{0}$ is of Hermitian type then $\Xi \simeq \mathcal{B} \times \overline{\mathcal{B}}$, see [7], Sect.3, [13], Prop. 2.2 , or [11], Prop. 6.1.9. The cycle space in that case was first described by J. Wolf and R. Zierau [39, 40]. Namely, in accordance with the above theorem, $\mathcal{M}_{D} \simeq \mathcal{B} \times \overline{\mathcal{B}}$ if $D$ is of non-holomorphic type and $\mathcal{M}_{D} \simeq \mathcal{B}$ or $\mathcal{M}_{D} \simeq \overline{\mathcal{B}}$ if $D$ is of holomorphic type. For $G_{0}$ of non-Hermitian type, the crucial equality $G\{D\}^{\circ}=\Omega$ is proved by G. Fels and A.T. Huckleberry in [10], see Thm. 4.2.5, using Kobayashi hyperbolicity of certain $G_{0}$-invariant domains in $G / K$. In the next section, we consider some properties of the crown $\Xi$, which are important for that proof and are interesting in themselves. After that, we explain the strategy of their proof, without going into the details.

Meanwhile, the notion of the cycle space has been generalized to lowerdimensional orbits and it turned out that its description in terms of the 
universal domain holds in this greater generality. Namely, given any $K$ orbit $O$ on $X=G / P$, S.G. Gindikin and T. Matsuki [13] defined a subset of $G$ by

$$
C(O)=\left\{g \in G \mid g O \cap O^{\prime} \neq \emptyset \text { and } g O \cap O^{\prime} \text { is compact }\right\},
$$

where $O^{\prime}$ is the corresponding $G_{0}$-orbit, i.e., $O \leftrightarrow O^{\prime}$. Let $C(O)^{\circ}$ be the connected component of $C(O)$ containing $e \in G$. Of course, if $D=O^{\prime}$ is open, then $C(O)=G\{D\}$ is the open set considered above. The following theorem was stated as a conjecture in [13], see Conjecture 1.6.

Theorem 7.2 (T. Matsuki [27]). Let $G, G_{0}$ and $X$ be as above. Then $C(O)^{\circ}=\Omega$ for all $K$-orbits on $X$ of non-holomorphic type.

Remark. The proof in [27] uses combinatorial description of the inclusion relations between the closures of $K$-orbits on the flag manifolds of $G$. As a corollary, we get that $C(O)^{\circ}$ is an open set, which is not clear a priori. If this is known, then Theorem 7.2 follows from [15] or from Theorem 12.1.3 in [11]. The latter asserts that the connected component of the interior of $C(O)$, containing the neutral element $e \in G$, coincides with $\Omega$.

\section{Complex geometric properties of the crown}

The following theorem proves the conjecture stated in [1].

Theorem 8.1 (D. Burns, S. Halverscheid, R. Hind [7]). The crown $\Xi$ is a Stein manifold.

The crucial ingredient of the proof is the construction of a smooth strictly plurisubharmonic function on $\Xi$ that is $G_{0}$-invariant and gives an exhaustion of the orbit space $G_{0} \backslash \Xi$. We call such a function a BHH-function. Let $\Gamma \subset G_{0}$ be a discrete cocompact subgroup acting freely on $G_{0} / K_{0}$. Then $\Gamma$ acts properly and freely on $\Xi$ and any BHH-function induces a plurisubharmonic exhaustion of $\Gamma \backslash \Xi$. Thus $\Gamma \backslash \Xi$ is a Stein manifold and its covering $\Xi$ is also Stein.

We now want to give another application of BHH-functions. Let $G_{0}=$ $K_{0} A_{0} N_{0}$ be an Iwasawa decomposition and let $B$ be a Borel subgroup of $G$ containing the solvable subgroup $A_{0} N_{0}$. Then $B$ is called an IwasawaBorel subgroup, the orbit $B(o) \subset G / K$ is Zariski open and its complement, to be denoted by $\mathcal{H}$, is a hypersurface. The set

$$
\Psi=\bigcap_{g \in G_{0}} g B(o)=\bigcap_{k \in K_{0}} k B(o)
$$

is open as the intersection of a compact family of open sets. Let $\Xi_{I}$ be the connected component of $\Psi$ containing $o$. L. Barchini [3] showed that $\Xi_{I} \subset \Xi$. The reverse inclusion was checked in many special cases including all classical groups and all real forms of Hermitian type, see [13, 19]. The 
proof in the general case is due to A. Huckleberry, see [14, 10] and [11], Remark 7.2.5. His argument is as follows. It is enough to prove that $\mathcal{H} \cap \Xi=\emptyset$. Assuming the contrary, observe that $\mathcal{H} \cap \Xi$ is $A_{0} N_{0}$-invariant and so $G_{0} \cdot(\mathcal{H} \cap \Xi)$ is closed in $\Xi$. Pick a BHH-function, restrict it to $\mathcal{H} \cap \Xi$ and take a minimum point $x_{*} \in \mathcal{H} \cap \Xi$ of the restriction. Then all points of the orbit $A_{0} N_{0}\left(x_{*}\right)$ are minimum points. Therefore $A_{0} N_{0}\left(x_{*}\right)$ is a totally real submanifold of dimension equal to $\operatorname{dim} G_{0} / K_{0}=\operatorname{dim}_{\mathbb{C}} G / K$ that is contained in $\mathcal{H}$, contrary to the fact that $\mathcal{H}$ is a proper analytic subset. From these considerations we get the following description of $\Xi$, see Theorem 8.2.

Remark. For a proof of the inclusion $\Xi \subset \Xi_{I}$ in a more general setting see [26]. Namely, the result is true for a connected real semisimple Lie group with two commuting involutions whose product is a Cartan involution. The corresponding fixed point subgroups generalize $G_{0}$ and $K$. The universal domain is defined similarly. The proof is based on a detailed study of double coset decompositions. Complex analytic techniques and, in particular, the existence of BHH-functions are not used.

Theorem 8.2. $\Xi=\Xi_{I}$.

Since $\Xi_{I}$ is a connected component of the open set $\Psi$, which is obtained by removing a family of hypersurfaces from the affine variety $G / K$, we see again that $\Xi$ is Stein. Since $\Psi$ is the set of all points for which the $k B k^{-1}$-orbit is open for every $k \in K_{0}$, we have

$$
\Psi=\left\{x \in G / K \mid \mathfrak{g}_{x}+(\operatorname{Ad} k) \cdot \mathfrak{b}=\mathfrak{g} \text { for all } k \in K_{0}\right\} .
$$

Let $N$ be the normalizer of $K$. Then $\Gamma=N / K$ is a finite group with a free action $x \rightarrow x^{\gamma}$ on $G / K$. From the last description of $\Psi$, it follows that $\Psi^{\gamma}=\Psi$ for all $\gamma \in \Gamma$. Thus $\Gamma$ interchanges the connected components of $\Psi$. It follows from the definition that $\Xi$ is contractible, so a non-trivial finite group cannot act freely on $\Xi$. Hence $\Gamma$ interchanges simply transitively the open sets $\Xi^{\gamma}$. Moreover, for any group $\tilde{K} \subset G$ with connected component $K$ the covering map $G / K \rightarrow G / \tilde{K}$ induces a biholomorphic map of $\Xi$ onto its image, cf. [11], Cor. 11.3.6.

Theorem 8.3 (A. Huckleberry [14]). $\Xi$ is Kobayashi hyperbolic.

Proof. By Frobenius reciprocity, there exist a $G$-module $V$ and a vector $v_{0} \in V$ such that $K \subset G_{v_{0}} \neq G$. If $G_{0}$ is of non-Hermitian type then $K$ is a maximal connected subgroup of $G$. If $G_{0}$ is of Hermitian type then there are precisely two intermediate subgroups between $K$ and $G$, both of them being parabolic. In any case, the connected component of the stabilizer of the line $\left[v_{0}\right]$ equals $K$ and the natural maps $G / K \rightarrow G v_{0} \rightarrow G\left[v_{0}\right]$ are finite coverings. Let $\mathbb{C}[V]_{d} \subset \mathbb{C}[V]$ be the subspace of homogeneous polynomials of degree $d$, let $I_{d}$ be the intersection of $\mathbb{C}[V]_{d}$ with the ideal of (the closure of) $G\left[v_{0}\right]$ and let $M_{d}$ be a $G$-stable complement to $I_{d}$ in $\mathbb{C}[V]_{d}$. The space of all polynomials in $M_{d}$ vanishing on $G\left[v_{0}\right] \backslash B\left[v_{0}\right]$ is $B$-stable and non-trivial for some $d$, so $B$ has an eigenvector $\varphi$ in that space. The zero set of $\varphi$ on the orbit $G\left[v_{0}\right]$ is exactly the complement to 
the open $B$-orbit $B\left[v_{0}\right]$. Replacing $V$ by its symmetric power $S^{k} V$ and $v_{0}$ by $v_{0}^{k} \in S^{k} V$, we obtain a linear form $\varphi$ with the same property. Now, let $V_{0}$ be the intersection of all hypersurfaces $g^{*} \varphi=0, g \in G$. Then $V_{0}$ is a $G$-stable linear subspace of $V$ and we have the $G$-equivariant linear projection map $\pi: V \rightarrow W=V / V_{0}$. Let $w_{0}=\pi\left(v_{0}\right)$ and let $\psi \in W^{*}$ be the linear form defined by $\pi^{*} \psi=\varphi$. Then $K \subset G_{w_{0}}$ and $G_{w_{0}} \neq G$, because $\varphi$ is non-constant on the orbit $G v_{0}$. Therefore $\pi$ gives rise to the finite coverings $G v_{0} \rightarrow G w_{0}$ and $G\left[v_{0}\right] \rightarrow G\left[w_{0}\right]$. By construction, the orbit $G \psi=\left\{g^{*} \psi \mid g \in G\right\}$ generates $W^{*}$ and the same is true for $G_{0} \psi$. By [14], Cor. 2.13, there exist hyperplanes $\mathcal{H}_{i}=\left\{g_{i}^{*} \psi=0\right\} \subset$ $\mathbb{P}(W), g_{i} \in G_{0}, i=1, \ldots, 2 m+1, m=\operatorname{dim} \mathbb{P}(W)$, satisfying the normal crossing conditions. It is then known that $\mathbb{P}(W) \backslash \bigcup_{i} \mathcal{H}_{i}$ is Kobayashi hyperbolic, see [17], Cor. 3.10.9. The intersection of this set with the orbit $G\left[w_{0}\right]$ equals $\bigcap_{i} g_{i} B\left[w_{0}\right]$ and is likewise hyperbolic. Recall that we have an equivariant fibering $G / K \rightarrow G / \tilde{K}=G\left[w_{0}\right]$. As we have seen before stating the theorem, $\Xi$ is mapped biholomorpically onto its image. The latter is contained in the connected component of $\bigcap_{i} g_{i} B\left[w_{0}\right]$ at $\left[w_{0}\right]$ and is therefore hyperbolic.

Theorem 8.4 (G. Fels, A. Huckleberry [10]). If $\Xi^{\prime}$ is a $G_{0}$-invariant, Stein, and Kobayashi hyperbolic domain in $G / K$ that contains $\Xi$ then $\Xi^{\prime}=\Xi$.

The proof requires analysis of the boundary bd $(\Xi)$. First, one considers the special case of $G_{0}=S L_{2}(\mathbb{R})$ and proves Theorem 8.4 for the crown $\Xi_{\mathfrak{s} l_{2}}$ of $S L_{2}(\mathbb{R}) / S O_{2}(\mathbb{R})$. Note that $G=S L_{2}(\mathbb{C})$ has precisely two nonisomorphic affine homogeneous surfaces. Namely, if $T \simeq \mathbb{C}^{*}$ is a maximal torus in $S L_{2}(\mathbb{C})$ and $N \subset S L_{2}(\mathbb{C})$ is the normalizer of $T$, then these surfaces are of the form $Q_{1}=S L_{2}(\mathbb{C}) / T \simeq\left(\mathbb{P}^{1}(\mathbb{C}) \times \mathbb{P}^{1}(\mathbb{C})\right) \backslash \Delta$ and $Q_{2}=S L_{2}(\mathbb{C}) / N \simeq \mathbb{P}^{2}(\mathbb{C}) \backslash C$, where $\Delta$ is the diagonal and $C$ is a nondegenerate curve of degree 2 . The crown $\Xi_{\mathfrak{s} l_{2}}$ can be viewed as a domain in $Q_{1}$ or in $Q_{2}$. In the general case one constructs a $G_{0}$-stable open dense subset bd $\left.\mathrm{bden}^{\text {gen }} \Xi\right) \subset \mathrm{bd}(\Xi)$, such that for $z \in \mathrm{bd}^{\text {gen }}(\Xi)$ there exists a simple 3-dimensional subalgebra $\mathfrak{s}_{0} \subset \mathfrak{g}_{0}$ with the following properties:

(i) the orbit of the corresponding complex group $S=\exp \left(\mathfrak{s}_{0}^{\mathbb{C}}\right) \subset G$ through $z$ is an affine surface, i.e., $S z \simeq Q_{1}$ or $S z \simeq Q_{2}$;

(ii) under this isomorphism $S z \cap \Xi$ is mapped biholomorphically onto $\Xi_{\mathfrak{s} l_{2}}$.

Now, if $\Xi^{\prime} \backslash \Xi \neq \emptyset$ then one can find a point $z$ as above in $\Xi^{\prime} \cap \operatorname{bd}(\Xi)$. Then $S z \cap \Xi^{\prime}$ properly contains $S z \cap \Xi$, contrary to the fact that $\Xi_{\mathfrak{s l} l_{2}}$ is a maximal $S L_{2}(\mathbb{R})$-invariant, Stein and Kobayashi hyperbolic domain in $Q_{1}$ or in $Q_{2}$. The details are found in [11], see Thm. 10.6.9.

Remark. In fact, $\Xi$ is the unique maximal $G_{0}$-invariant, Stein, and Kobayashi hyperbolic domain in $G / K$ that contains the base point $o$, see [11], Thm. 11.3.1.

Remark. We refer the reader to [12] for the definition of the Shylov-type 
boundary of the crown and to [20] for its simple description and applications to the estimates of automorphic forms.

\section{The Schubert domain}

We assume here that $G_{0}$ is of non-Hermitian type. Then the map $G / K \rightarrow$ $G / L$ is a finite covering. We have an open $G_{0}$-orbit $D \subset X=G / P$ and the corresponding compact $K$-orbit $C_{0} \subset D$. Let $q$ denote the complex dimension of $C_{0}$. Translations $g C_{0}, g \in G$, are called cycles and are regarded as points of $\mathcal{M}_{X}:=G / L$. The cycle space $\mathcal{M}_{\mathcal{D}}$ is a domain in $\mathcal{M}_{X}$ and the crown $\Xi$ is mapped biholomorphically onto a domain $\tilde{\Xi} \subset \mathcal{M}_{X}$. We want to prove the statement of Theorem 7.1, namely, that $G\{D\}^{\circ}$ agrees with $\Omega$. Equivalently, we will prove that $\mathcal{M}_{\mathcal{D}}$ agrees with $\tilde{\Xi}$. A. Huckleberry and J. Wolf [16] defined the Schubert domain $\mathcal{S}_{D}$ in $\mathcal{M}_{X}$ as follows. Let $B$ be an Iwasawa-Borel subgroup of $G$. The closures of $B$-orbits on $X$ are called Schubert varieties (with respect to $B$ ). The group $B$ has an open orbit on any such variety $S$. Since the open orbit is affine, its complement $S^{\prime}$ is a hypersurface in $S$. For topological reasons, the (finite) set $\mathcal{S}_{C_{0}}$ of Schubert varieties of codimension $q$ intersecting $C_{0}$ is non-empty. One shows that $S^{\prime} \cap D=\emptyset$ for any $S \in \mathcal{S}_{C_{0}}$. Thus the incidence variety

$$
\mathcal{H}(S):=I\left(S^{\prime}\right)=\left\{g C_{0} \in \mathcal{M}_{X} \quad \mid g C_{0} \cap S^{\prime} \neq \emptyset\right\}
$$

is contained in $\mathcal{M}_{X} \backslash \mathcal{M}_{D}$. Clearly, $\mathcal{H}(S)$ is $B$-invariant. Furthermore, one can show that $\mathcal{H}(S)$ is an analytic hypersurface in $\mathcal{M}_{X}$, see [11], Prop.7.4.11. For any $k \in K_{0}$ we have $\mathcal{M}_{D} \subset \mathcal{M}_{X} \backslash k \mathcal{H}(S)$. The set

$$
\bigcup_{S \in \mathcal{S}_{C_{0}}}\left\{\bigcup_{k \in K_{0}} k \mathcal{H}(S)\right\}
$$

is closed in $\mathcal{M}_{X}$. Its complement is denoted by $\mathcal{S}_{D}$ and is called the Schubert domain. By construction, $\mathcal{S}_{D}$ is a $G_{0}$-invariant Stein domain and

$$
\mathcal{M}_{D} \subset \mathcal{S}_{D}
$$

On the other hand, for any boundary point $z \in \operatorname{bd}(D)$ there exist an Iwasawa decomposition $G_{0}=K_{0} A_{0} N_{0}$, an Iwasawa-Borel subgroup $B$ containing $A_{0} N_{0}$ and a $B$-invariant variety $S_{z}$ of codimension $q+1$, such that $z \in S_{z}$ and $D \cap S_{z}=\emptyset$ (a supporting Schubert variety at $z$ ), see [11], Prop. 9.1.2. Take a boundary point of $\mathcal{M}_{D}$ and consider the corresponding cycle. It has a point $z \in \operatorname{bd}(D)$, hence is contained in the incidence variety

$$
I\left(S_{z}\right):=\left\{g C_{0} \mid g C_{0} \cap S_{z} \neq \emptyset\right\} .
$$

Obviously, $I\left(S_{z}\right)$ is $B$-invariant and $I\left(S_{z}\right) \subset \mathcal{M}_{X} \backslash \mathcal{M}_{D}$, in particular, $I\left(S_{z}\right) \neq \mathcal{M}_{X}$. But $\tilde{\Xi}$ is contained in the open $B$-orbit by Theorem 8.2. Thus a point of $\tilde{\Xi}$ cannot be a boundary point of $\mathcal{M}_{D}$, and it follows that

$$
\tilde{\Xi} \subset \mathcal{M}_{D} .
$$


Finally, one can modify the proof of Theorem 8.3 to show that $\mathcal{S}_{D}$ is hyperbolic. Namely, take the linear bundle $\mathbb{L}$ over $G / L$ defined by the hypersurface $\mathcal{H}(S)$, which appears in the definition of $\mathcal{S}_{D}$. Then some power $\mathbb{L}^{k}$ admits a $G$-linearization. Thus we obtain a non-degenerate equivariant map $G / L \rightarrow \mathbb{P}(W)$, where a $G$-module $W$ is generated by a weight vector of $B$. The map is in fact a finite covering over the image, which is a $G$-orbit in $\mathbb{P}(W)$ containing the image of $\mathcal{H}(S)$ as a hyperplane section. Since $W$ is irreducible, the same argument as in the proof of Theorem 8.3 shows that $\mathcal{S}_{D}$ is hyperbolic. The inclusions $(*)$ and $(* *)$, together with Theorem 8.4, imply

$$
\tilde{\Xi}=\mathcal{M}_{D}=\mathcal{S}_{D} .
$$

\section{Complex geometric properties of flag domains}

An open $G_{0}$-orbit in a complex flag manifold $X=G / P$ is called a flag domain. One classical example of a flag domain is a bounded symmetric domain in the dual compact Hermitian symmetric space. In this example, a flag domain is a Stein manifold. However, this is not the case for an arbitrary flag domain $D$, because $D$ may contain compact complex submanifolds of positive dimension. As we have seen, the cycle space of $D$ is always Stein. Here, we consider the properties of $D$ itself.

An open orbit $D=G_{0}\left(x_{0}\right) \subset X$ is said to be measurable if $D$ carries a $G_{0}$-invariant volume element. We retain the notation of Section 2 . In particular, $x_{0}=e \cdot P, \mathfrak{p}=\mathfrak{p}_{\Phi}$, where $\mathfrak{p}$ and $\Phi$ satisfy (i), (ii) of Theorem 2.4 .

Theorem 10.1 (J. Wolf [38], Thm. 6.3). The open orbit $G_{0}\left(x_{0}\right)$ is measurable if and only if $\tau \Phi^{r}=\Phi^{r}$ and $\tau \Phi^{u}=-\Phi^{u}$. Equivalently, $G_{0}\left(x_{0}\right)$ is measurable if and only if $\mathfrak{p} \cap \tau \mathfrak{p}$ is reductive.

Since two fundamental Cartan subalgebras in $\mathfrak{g}_{0}$ are conjugate by an inner automorphism of $G_{0}$, it follows from the above condition and from Theorem 2.4 that all open $G_{0}$-orbits on $X$ are measurable or non-measurable simultaneously. The proof of Theorem 10.1 can be also found in [11], Sect. 4.5 .

Example 10.2. Let $\mathfrak{g}_{0}$ be a real form of inner type. Since the Cartan subalgebra $\mathfrak{t} \subset \mathfrak{g}$ contains a compact Cartan subalgebra $\mathfrak{j} \subset \mathfrak{g}_{0}$, it follows that $\tau(\alpha)=-\alpha$ for any root $\alpha$. Thus the open orbit $G_{0}\left(x_{0}\right)$ is measurable.

Example 10.3. If $P=B$ is a Borel subgroup of $G$, then $\Phi=\emptyset, \Phi^{u}=\Sigma^{+}$ and $\tau \Phi^{u}=-\Phi^{u}$. Therefore an open $G_{0}$-orbit in $G / B$ is measurable.

A complex manifold $M$ is said to be $q$-complete if there is a smooth nonnegative exhaustion function $\varrho: M \rightarrow \mathbb{R}$, whose Levi form has at least $n-q$ positive eigenvalues at every point of $M$. A fundamental theorem 
of A. Andreotti and H. Grauert says that for any coherent sheaf $\mathcal{F}$ on a $q$-complete manifold and for all $k>q$ one has $H^{k}(M, \mathcal{F})=0$, see [2]. Note that in the older literature including [2] the manifolds that we call $q$-complete were called $(q+1)$-complete.

Theorem 10.4 (W. Schmid, J. Wolf [33]). If $D$ is a measurable open $G_{0}$-orbit in a flag manifold of $G$ and $q$ is the dimension of the compact $K$-orbit in $D$ then $D$ is $q$-complete. In particular, $H^{k}(D, \mathcal{F})=0$ for all coherent sheaves on $D$ and for all $k>q$.

The authors of [33] do not say that $D$ is measurable, but they use the equivalent condition that the isotropy group of $D$ is the centralizer of a torus. The proof of Theorem 10.4 can be also found in [11], see Thm. 4.7.8.

Example 10.5. Let $X=\mathbb{P}^{n}(\mathbb{C}), G=S L_{n+1}(\mathbb{C})$, and $G_{0}=S L_{n+1}(\mathbb{R})$. Let $\left\{e_{1}, e_{2}, \ldots, e_{n+1}\right\}$ be a basis of $\mathbb{R}^{n+1}$. If $n>1$ then $G_{0}$ has two orbits on $X$, the open one and the closed one, with representatives $x_{0}=\left[e_{1}+i e_{2}\right]$ and $\left[e_{1}\right]$, respectively. The isotropy subgroup $\left(G_{0}\right)_{x_{0}}$ is not reductive. Its unipotent radical consists of all $g \in G_{0}$, such that

$$
g\left(e_{i}\right)=e_{i}(i=1,2), g\left(e_{j}\right) \equiv e_{j} \bmod \left(\mathbb{R} e_{1}+\mathbb{R} e_{2}\right)(j \geq 3) .
$$

Hence the open orbit $D=G_{0}\left(x_{0}\right)=\mathbb{P}^{n}(\mathbb{C}) \backslash \mathbb{P}^{n}(\mathbb{R})$ is not measurable. Note that $K=S O_{n+1}(\mathbb{C})$. Thus the compact $K$-orbit $C_{0} \subset D$ is the projective quadric $z_{1}^{2}+z_{2}^{2}+\ldots+z_{n+1}^{2}=0$ and its dimension equals $n-1$. In this case, we have $n-q=1$ and we show how to construct a smooth non-negative exhaustion function $\varrho: \mathbb{P}^{n}(\mathbb{C}) \backslash \mathbb{P}^{n}(\mathbb{R}) \rightarrow \mathbb{R}$, whose Levi form has at least one positive eigenvalue at every point. For $z=x+i y \in \mathbb{C}^{n+1}$ put

$$
\varrho_{1}(z)=\sum x_{k}^{2}+\sum y_{k}^{2}, \quad \varrho_{2}(z)=\sqrt{\sum\left(x_{k} y_{l}-x_{l} y_{k}\right)^{2}}
$$

and notice that

$$
\varrho_{1}(\zeta z)=|\zeta|^{2} \varrho_{1}(z), \quad \varrho_{2}(\zeta z)=|\zeta|^{2} \varrho_{2}(z) \text { for any } \zeta \in \mathbb{C}^{*} .
$$

Thus

$$
\varrho([z])=\frac{\varrho_{1}(z)}{\varrho_{2}(z)}
$$

is well-defined for all $[z] \in \mathbb{P}^{n}(\mathbb{C}) \backslash \mathbb{P}^{n}(\mathbb{R})$. Obviously, $\varrho$ is a smooth exhaustion function for $\mathbb{P}^{n}(\mathbb{C}) \backslash \mathbb{P}^{n}(\mathbb{R})$. Given a point $[z]=[x+i y] \in$ $\mathbb{P}^{n}(\mathbb{C}) \backslash \mathbb{P}^{n}(\mathbb{R})$, take the line $L$ in $\mathbb{P}^{n}(\mathbb{C})$, connecting $[z]$ with $[x] \in \mathbb{P}^{n}(\mathbb{R})$, and restrict $\varrho$ to that line. Clearly, $L$ is the projective image of the affine line

$$
\lambda=\alpha+i \beta \mapsto w=x+i \lambda y=x-\beta y+i \alpha y
$$

and the restriction $\left.\varrho\right|_{L}$ equals

$$
\varphi(\lambda):=\varrho([w])=\frac{|\alpha|}{D} \sum y_{k}^{2}+\frac{1}{|\alpha| D} \sum\left(x_{k}-\beta y_{k}\right)^{2},
$$


where $D=\varrho_{2}(x+i y)$. Computing the Laplacian

$$
\Delta \varphi=\frac{\partial^{2} \varphi}{\partial \alpha^{2}}+\frac{\partial^{2} \varphi}{\partial \beta^{2}}
$$

for $\alpha \neq 0$, we get

$$
\Delta \varphi=\frac{2}{D|\alpha|^{3}} \sum\left(x_{k}-\beta y_{k}\right)^{2}+\frac{2}{D|\alpha|} \sum y_{k}^{2}>0,
$$

showing that $\varphi$ is strictly subharmonic. Hence the Levi form of $\varrho$ has at least one positive eigenvalue at $[z]=\left.[w]\right|_{\alpha=1, \beta=0}$.

Concluding remark. The open orbit in the last example is not measurable. As a matter of fact, the conclusion of Theorem 10.4 holds true in this case. In general, the author does not know whether one can drop the measurability assumption in Theorem 10.4.

\section{References}

[1] D.N. Akhiezer, S.G. Gindikin, On Stein extensions of real symmetric spaces, Math. Ann. 286 (1990), 1 - 12.

[2] A. Andreotti, H. Grauert, Théorèmes de finitude pour la cohomologie des espaces complexes, Bull. Soc. Math. France 90 (1962), 193 - 259.

[3] L. Barchini, Stein extensions of real symmetric spaces and the geometry of the flag manifold, Math. Ann. 326 (2003), 331 - 346.

[4] A. Borel, J. Tits, Éléments unipotents et sous-groupes paraboliques de groupes réductifs I, Invent. Math. 12, 2 (1971), 95 - 104.

[5] R. Bremigan, J. Lorch, Orbit duality for flag manifolds, Manuscripta Math. 109, 2 (2002), 233 - 261.

[6] M. Brion, Quelques propriétés des espaces homogènes sphériques, Manuscripta Math. 55, 2 (1986), 191 - 198

[7] D. Burns, S. Halverscheid, R. Hind, The geometry of Grauert tubes and complexification of symmetric spaces, Duke Math. J., 118, 3 (2003), 465 - 491.

[8] C. Chevalley, Théorie des groupes de Lie: groupes algébriques, théorèmes généraux sur les algèbres de Lie, Paris, Hermann, 1951.

[9] A. Dold, Lectures on algebraic topology, Springer, Berlin - Heidelberg - New York, second edition, 1980.

[10] G. Fels, A.T. Huckleberry, Characterization of cycle domains via Kobayashi hyperbolicity, Bull. Soc. Math. France, 133, 1 (2005), 121 -144 .

[11] G. Fels, A.T. Huckleberry, J.A. Wolf, Cycle spaces of flag domains (a complex analytic viewpoint), Progress in Mathematics, vol. 245, Birkhäuser, Boston - Basel - Berlin, 2006. 
[12] S. Gindikin, B. Krötz, Complex crowns of Riemannian symmetric spaces and non-compactly causal symmetric spaces, Trans. Amer. Math. Soc. 354, 8 (2002), 3299 - 3327.

[13] S. Gindikin, T. Matsuki, Stein extensions of Riemannian symmetric spaces and dualities of orbits on flag manifolds, Transform. Groups $\mathbf{8}, 4$ (2003), 333 - 376.

[14] A. Huckleberry, On certain domains in cycle spaces of flag manifolds, Math. Ann. 323 (2002), 797 - 810.

[15] A. Huckleberry, B. Ntatin, Cycle spaces of $G$-orbits in $G^{\mathbb{C}}$-manifolds, Manuscripta Math. 112, 4 (2003), 433 - 440.

[16] A. Huckleberry, J.A. Wolf, Schubert varieties and cycle spaces, Duke Math. J., 120, 2 (2003), 229 - 249.

[17] S. Kobayashi, Hyperbolic complex spaces, Springer-Verlag, Berlin Heidelberg, 1998.

[18] B. Kostant, On the conjugacy of real Cartan subalgebras I, Proc. Nat. Acad. Sci U.S.A. 41, 11 (1955), 967 - 970.

[19] B. Krötz, R. Stanton, Holomorphic extension of representations: (I) automorphic functions, Ann. of Math. 159, 2 (2004), 641 - 724.

[20] B. Krötz, E. Opdam, Analysis on the crown domain, Geom. Funct. Anal. 18, 4 (2008), 1326 - 1421.

[21] D. Luna, Slices étales, Bull. Soc. Math. France, Mémoire 33 (1973), $81-105$.

[22] F.M. Malyshev (Malyšev), Complex homogeneous spaces of semisimple Lie groups of the first category, Izv. Akad. Nauk SSSR, ser. mat. 39, 5 (1975), 992 - 1002, English transl.: Math. USSR Izv. 9, 5 (1975), 939 - 949.

[23] F.M. Malyshev (Malyšev), Complex homogeneous spaces of semisimple Lie groups of type $D_{n}$, Izv. Akad. Nauk SSSR, ser. mat. 41, 4 (1977), 829 - 852, English transl.: Math. USSR Izv. 11, 4 (1977), 783 -805 .

[24] T. Matsuki, Orbits on affine symmetric spaces under the action of parabolic subgroups, Hiroshima Math. J. 12, 2 (1982), 307 - 320.

[25] T. Matsuki, Closure relations for orbits on affine symmetric spaces under the action of parabolic subgroups. Intersections of associated orbits, Hiroshima Math. J. 18, 1 (1988), 59 - 67.

[26] T. Matsuki, Stein extensions of Riemann symmetric spaces and some generalization, J. Lie Theory 13, 2 (2003), 563 - 570.

[27] T. Matsuki, Equivalence of domains arising from duality of orbits on flag manifolds I, II, III, Trans. Amer. Math. Soc. 358, 5 (2005), 2217 - 2245; Proc. Amer. Math. Soc. 134, 12 (2006), 3423 - 3428; Trans. Amer. Math. Soc. 359, 10 (2007), 4773 - 4786.

[28] I. Mirković, T. Uzawa, K. Vilonen, Matsuki correspondence for sheaves, Invent. Math. 109, 2 (1992), 231 - 245. 
[29] D. Montgomery, Simply connected homogeneous spaces, Proc. Amer. Math. Soc. 1 (1950), 467 - 469.

[30] A.L. Onishchik, Inclusion relations among transitive compact transformation groups, Trudy Mosk. Mat. Obshch. 11 (1962), 199 - 242, English transl.: Amer. Math. Soc. Transl. (2) 50 (1966), 5 - 58.

[31] A.L. Onishchik, Decompositions of reductive Lie groups, Mat. Sbornik 80 (122) (1969), 553 - 599, English transl.: Math. USSR Sb. 9 (1969), 515 - 554.

[32] A.L. Onishchik, Topology of transitive transformation groups, J.A.Barth, Leipzig - Berlin - Heidelberg, 1994.

[33] W. Schmid, J. Wolf, A vanishing theorem for open orbits on complex flag manifolds, Proc. Amer. Math. Soc. 92, 3 (1984), 461 - 464.

[34] M. Sugiura, Conjugate classes of Cartan subalgebras in real semisimple Lie algebras, J. Math. Soc. Japan 11, 4 (1959), 374 - 434.

[35] E.B. Vinberg, Complexity of actions of reductive groups, Functional Analysis and Appl. 20, 1 (1986), 1 - 11.

[36] B. Weisfeiler, On one class of unipotent subgroups of semisimple algebraic groups, arXiv: math/0005149v1 [math. AG], translated from Russian: Uspekhi Mat. Nauk 21, 2 (128) (1966), 222 - 223.

[37] R.O. Wells, Jr., J.A. Wolf, Poincaré series and automorphic cohomology on flag domains, Ann. Math. 105, 3 (1977), 397 - 448.

[38] J.A. Wolf, The action of a real semisimple Lie group on a complex flag manifold. I: Orbit structure and holomorphic arc components, Bull. Amer. Math. Soc. 75, 6 (1969), 1121 - 1237.

[39] J.A. Wolf, R. Zierau, Linear cycle spaces in flag domains, Math. Ann. 316 (2000), 529 - 545.

[40] J.A. Wolf, R. Zierau, A note on the linear cycle space for groups of Hermitian type, J. Lie Theory 13, 1 (2003), 189 - 191.

D.N.Akhiezer

Institute for Information Transmission Problems,

19 B.Karetny per., Moscow, 127994, Russia.

Email: akhiezer@iitp.ru 